\title{
Steady Solutions of the Boltzmann Equation for a Gas Flow past an Obstacle, II. Stability
}

Dedicated to Professor Mizohata on his 60th anniversary

By

Seiji UkaI* and Kiyoshi AsAno**

\section{§1. Fintroduction}

This is a continuation of our previous work [9] on the exterior problem for the Boltzmann equation describing a gas flow past an obstacle. In [9], we established the existence of stationary solutions, and the subject of this paper is their stability.

The paper [9] will be referred to as Part $\mathbb{I}$ and any formulae number of Part I will be preceded by the symbol I, when quoted in this paper. Thus, (I.1.1a) means Equation (1.1a) of Part $\mathbb{I}$, and so on.

Our problem is the initial boundary value problem (I.1.1):

$$
\begin{array}{ll}
f_{t}=-\xi \cdot \nabla_{x} f+Q[f, f], & (t, x, \xi) \in \mathbb{R}_{+} \times \Omega \times \mathbb{R}^{n}, \\
r^{-} f=M r^{+} f, & (t, x, \xi) \in \mathbb{R}_{+} \times S^{-}, \\
f \rightarrow g_{c}(\xi)(|x| \rightarrow \infty), & (t, \xi) \in \mathbb{R}_{+} \times \mathbb{R}^{n}, \\
\left.f\right|_{t=0}=f^{0}, & (x, \xi) \in \Omega \times \mathbb{R}^{n} .
\end{array}
$$

For the notations, we refer the readers to Part $\mathbb{I}$. The stationary problem induced from (1.1) is (I.1.10). Let $u=u(c)$ be the solution obtained in Theo. rem I.9.3 to (I.1.12). Then,

$$
f_{c}=g_{0}+g_{c}^{1 / 2} u(c)
$$

is a (unique) solution to (I.1.10). The aim of the present paper is to show that $f_{c}$ is asymptotically stable in $t$. In other words, we shall show that whenever the initial $f^{0}$ in (1.1) is close to $f_{c}$, a unique solution $f=f(t)$ exists to (1.1) in the large in $t$ and approaches $f_{c}$ as $t \rightarrow \infty$.

Communicated by S. Matsuura, October 26, 1985.

* Department of Applied Physics, Osaka City University, Osaka 558, Japan.

** Institute of Mathematics, Yoshida College, Kyoto University, Kyoto 606, Japan. 
In order to prove this, we seek a solution $f$ of the form

$$
f=f(t)=f_{c}+g_{c}^{1 / 2} w(t)=g_{0}+g_{c}^{1 / 2}(u(c)+w(t)) .
$$

Substract (I.1.10) from (1.1) and recall the definition (I.1.11) of $L_{c}, \Gamma_{0}$ and $\tilde{M}_{0}$. Then, $w=w(t)$ should solve,

$$
\begin{aligned}
& w_{t}=-\xi_{\circ} \nabla_{x} w+L_{c} w+2 \Gamma_{0}[u(c), w]+\Gamma_{0}[w, w], \\
& \tilde{M}_{0} w=0, \\
& w \rightarrow 0(|x| \rightarrow \infty), \\
& \left.w\right|_{t=0}=w_{0} .
\end{aligned}
$$

Obviously, the desired stability of $f_{c}$ follows if for each small $w_{0},(1.2)$ has a unique solution in the large in $t$ tending to 0 as $t \rightarrow \infty$.

This has been shown in [1] for the special case $c=0$ in which $u(0)=0$ (see Theorem I.9.3 (i)). The method employed there is based on the contraction mapping principle supplemented by nice decay estimates of the semigroup for the linearized equation of (1.2). This method is the only possible method, at least up to the present, which makes accessible the nonlinear Boltzmann equation in the large in $t$, see $[2,4-8]$.

To apply it to (1.2) for the case $c \neq 0$, recall the operator $B_{c}$ of (I.1.13). It is the linear operator

$$
B_{c}=\xi \circ \nabla_{x}+L_{c}, \quad(x, \xi) \in \Omega \times \mathbb{R}^{n},
$$

associated with the boundary condition $\tilde{M}_{0} w=0$ on $S^{-}$and $w \rightarrow 0(|x| \rightarrow \infty)$. It was shown in $\S \mathbb{I}$.7 that $B_{c}$ generates a semigroup in $L^{2}\left(\Omega \times \mathbb{R}^{n}\right)$. Denote the semigroup by $\mathbb{E}_{c}(t)$;

$$
\mathbb{E}_{c}(t)=\exp \left(t B_{c}\right)
$$

Then, (1.2) is reduced to the integral equation,

$$
w(t)=E_{c}(t) w_{0}+\int_{0}^{t} E_{c}(t-s)\left\{2 \Gamma_{0}[u(c), w(s)]+\Gamma_{0}[w(s), w(s)]\right\} d s .
$$

In $\S 5$, we will solve (1.4) in the large in $t$ by the help of the contraction mapping principle. Then, a decay estimate of $E_{c}(t)$ is needed, and will be discussed in $\S 4$ by a perturbation argument based on the explicit expression of the resolvent of $B_{c}$ found in $\S \mathbb{1}$.7. The unperturbed operator is $B_{c}^{\infty}$ of $\S \mathbb{I} .6$, i.e., (1.3) defined for $(x, \xi) \in \mathbb{R}^{n} \times \mathbb{R}^{n}$. We shall discuss the semigroup $E_{c}^{\infty}(t)$ generated by $B_{c}^{\infty}$ in $\S 3 . \quad \S 2$ is a preliminary and $\S 6$ gives the proof of Proposition 4.5 which is a key to $\S 4$. As announced in $\mathbb{P a r t} \mathbb{I}$, the proof of 
Proposition I.7.4 which plays an essential role in $\S 6$ as well as $\S \mathbb{1}$.7 will be given in $\$ 7$.

Observe that the linear operator associated to (1.2) is not $B_{c}$ but $B_{c}+2 \Gamma_{0}$ $[u(c), \circ]$, and that if the semigroup for the latter is used, then the linear term on the right hand side of (1.4) is absent. However, our perturbation technique no longer works to get any decay estimates for the latter semigroup, so, only $E_{c}(t)$ is available. On the other hand, the extra linear term on the right hand side of (1.4) can be handled only by the decay estimate of $E_{c}(t)$ sharper than recuired when the linear term is absent; we need the estimate $E_{c}(t)=0\left(t^{-\infty}\right), \alpha>1$ for (1.4) while $\alpha>1 / 2$ suffices if the linear term is absent. Also, to handle the linear term, we are required that $u(c)$ is small, namely, $c$ is small.

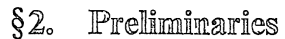

Given a Banach space $X$, we define the Banach space $X_{\omega}, \alpha \in \mathbb{R}$, of functions $f(t)$ of $t \geq 0$ with values in $X$ by

$$
f(t) \in x_{\omega} \Leftrightarrow(1+\hat{\imath})^{\alpha} f(t) \in \mathbb{L}^{\infty}([0, \infty) ; X)
$$

When $X$ is any one of the spaces $L_{\beta}^{p, q}(Q), Y_{\beta}^{p, q_{,} \pm}$, etc. of $\mathbb{P a r t} \mathbb{I}$, we write $L_{\beta, \alpha}^{p, q}(Q)$, $\mathbb{Y}_{\beta, \alpha}^{p, q, \pm}$, etc. for $X_{\alpha}$.

Let $Y$ be another Banach space and $\mathbb{B}(X, Y)$ denote the space of all bounded linear operators from $Y$ io $Y$. Then $\mathbb{B}_{a}(X, Y)$ will be defined by (2.1) with $\mathbb{B}(X, Y)$ substituted for $X$. If $A(t) \in \mathbb{B}_{\alpha}(X, Y)$, it induces the multiplication operator $A$,

$$
(A u)(t)=A(t) u, \quad u \in \mathbb{X},
$$

and the convolution operator $A *$,

$$
(A * f)(t)=\int_{0}^{t} A(t-s) f(s) d s .
$$

The following two lemmas will be userul in the sequel.

Lemma 2. H。 Let $A(t) \in \mathbb{B}_{\alpha}(X, Y)$.

(i) $A \in \mathbb{B}\left(\mathbb{X}, Y_{\alpha}\right)$.

(ii) Suppose $\alpha>0$. For each $\gamma>0$, put $\mu=\min (\alpha, \gamma, \alpha+\gamma-1)$.

If $\alpha \neq 1$ or $\gamma \neq 1$, then

$$
A * \in \mathbb{B}\left(X_{\gamma}, \mathbb{Y}_{\mu}\right)
$$


If $\alpha>1$ and $\gamma \in[0, \alpha]$, then $\mu=\gamma$.

Proof. (i) is obvious and (ii) is a consequence of

$$
\int_{0}^{t}(1+t-s)^{-\infty}(1+s)^{-\gamma} d s \leq C(1+t)^{-\mu}, \quad t \geq 0,
$$

which can be shown splitting the integral over $(0, t / 2)$ and $(t / 2, t)$.

Lemma 2.2. Suppose $A(t) \in L^{\infty}([\varepsilon, \infty) ; \boldsymbol{B}(X, Y))$ for all $\varepsilon>0$ and satisfy

$$
\|A(t)\|_{B(X, Y)} \leq C t^{-\delta} e^{-\nu t}, \quad t>0,
$$

with some constants $C \geq 0, \delta<1$ and $\nu>0$. Then for all $\alpha \geq 0$,

$$
A * \in \mathbb{B}\left(X_{\alpha}, Y_{\alpha}\right) \text {. }
$$

Proof. This is immediate from

$$
\int_{0}^{t}(t-s)^{-\delta} e^{-\nu(t-s)}(1+s)^{-\alpha} d s \leq C \nu^{\delta-1}\left(1+\nu^{-\alpha}\right)(1+t)^{-\alpha}, \quad t \geq 0 .
$$

For a function $f(t) \in X$ of $t \geq 0$, the Laplace transform $\hat{f}=\mathcal{L}_{t} f$ is defined by

$$
\hat{f}(\lambda)=\left(\mathcal{L}_{t} f\right)(\lambda)=\int_{0}^{\infty} e^{-\lambda t} f(t) d t
$$

If $e^{-\sigma t} f(t) \in X_{\alpha}$ with some $\alpha, \sigma \in \mathbb{R}$, then $\hat{f}(\lambda) \in X$ exists for all $\lambda \in \mathbb{C}_{+}(\sigma)$ and is analytic in $\lambda$. If $f_{1}(t), f_{2}(t)$ are two such functions, then

$$
\left(\mathcal{L}_{t} f_{1} * f_{2}\right)(\lambda)=\hat{f}_{1}(\lambda) \hat{f}_{2}(\lambda)
$$

holds for all $\lambda \in \boldsymbol{C}_{+}(\sigma)$.

For a function $g(\lambda) \in X$ of $\lambda \in C_{+}(\sigma)$, the inverse Laplace transform $\stackrel{\mathrm{g}}{=}=\mathcal{L}_{t}^{-1} g$ is defined by

$$
\stackrel{y}{g}(t)=s-\lim _{a \rightarrow \infty} \frac{1}{2 \pi i} \int_{\eta+i a}^{\eta+i a} e^{\lambda t} g(\lambda) d \lambda, \quad \eta>\sigma,
$$

the topology of the convergence being that of $X$. The following simple sufficient condition for $g(\lambda)$ to be a Laplace transform will be used later.

Lemma 2.3. Let $g(\lambda) \in X$ be defined and analytic in $\lambda \in \bar{C}_{+}(\sigma)$. Suppose

$$
\begin{aligned}
& \sup _{\tau \in \mathbb{R}}\|g(\eta+i \tau)\|_{X} \rightarrow 0(\eta \rightarrow \infty), \\
& \sup _{\eta \geq \sigma}\|g(\eta+i \tau)\|_{X} \leq C(1+|\tau|)^{-\delta}, \quad \tau \in \mathbb{R}
\end{aligned}
$$


with some constants $C \geq 0$ and $\delta>1$. Then there exists a unique $\stackrel{g}{g}(t)$ such that $e^{-\sigma t} \stackrel{v}{g}(t) \in \mathscr{B}^{0}([0, \infty) ; X)$, and for all $\lambda \in \mathbb{C}_{+}(\sigma)$, there holds

$$
g(\lambda)=\left(\mathcal{L}_{t} \stackrel{\stackrel{g}{g}}{)}(\lambda)\right.
$$

Obviously, the above result remains valid if $X$ is replaced by $\mathbb{B}(X, Y)$.

\section{§3. Asymptotic Behaviors of $\mathbb{E}_{c}^{\infty}(\mathbb{E})$}

Recall the Definition (I.6.1) of the operator $B_{c}^{\infty}$. As shown there, it generates a strongly continuous semigroup in the space $L^{2}\left(Q^{\infty}\right), Q^{\infty}=\mathbb{R}_{x}^{n} \times \mathbb{R}_{\xi}^{n}$. Denote this semigroup as $E_{c}^{\infty}(t)$. According to the convention stated in $\S I$. $E_{c}^{\infty}(t)$ will also express its realizations in other spaces.

Let $\widetilde{L}_{\beta}^{p, q}\left(Q^{\infty}\right)$, etc., be the spaces defined in $\S \mathbb{I} .2$. In this section we write

$$
\begin{array}{ll}
\widetilde{L}_{\beta}^{p, q}=\widetilde{L}_{\beta}^{p, q}\left(Q^{\infty}\right), & \widetilde{L}_{\beta, \alpha}^{p, q}=\widetilde{L}_{\beta, \alpha}^{p, q}\left(Q^{\infty}\right), \\
Z^{q}=\widetilde{L}_{0}^{2,2} \cap \widetilde{L}_{0}^{q, 2}, & Z_{\alpha}^{q}=\widetilde{L}_{0, \alpha}^{2,2} \cap \widetilde{L}_{0, \alpha}^{q, 2} .
\end{array}
$$

The constants $C \geq 0$ and operator norms appearing in the followings are all locally bounded in $c \in \mathbb{R}^{n}$ and possibly independent of $t$.

Let $P_{c}$ be the projection of Lemma I.3.8 and let $\Lambda_{c}^{\delta}=\nu_{c}(\xi)^{\delta} \times$. The main result of this section is as follows.

Theorem 3.1. Let $1 \leq q \leq 2 \leq p \leq \infty, \beta \in \mathbb{R}, m=0,1$ and put $\alpha=$ $\frac{n}{2}\left(\frac{1}{q}-\frac{1}{p}\right)+\frac{m}{2}$.

(i) There is a constant $C \geq 0$ such that

$$
\left\|E_{c}^{\infty} *\left(I-P_{c}\right)^{m} u\right\|_{\tilde{L}_{\beta, \infty}^{p, \infty}} \leq C\left(\|u\|_{\tilde{L}_{\beta}^{p, \infty}}+\|u\|_{Z^{q}}\right) .
$$

(ii) Let $r \geq 0$ and $\delta \leq 1$, and put $\mu=\min (\alpha, \gamma, \alpha+\gamma-1)$. Then there is a constant $C \geq 0$ such that if $\gamma \neq 1$,

$$
\left\|E_{c}^{\infty} *\left(I-P_{c}\right)^{m} \Lambda_{c}^{\delta} f\right\|_{\tilde{L}_{\beta, \mu}^{p, \infty}} \leq C\left(\|f\|_{\tilde{L}_{\beta, \gamma}^{p, \infty}}+\left\|\Lambda_{c}^{\delta} f\right\|_{Z_{\gamma}^{q}}\right) .
$$

This theorem for the case $c=0$ has been established essentially in [4] and [6]. Since the case $c \neq 0$ can be dealt with in the same way, only the outline of the proof will be presented.

Let us begin by recalling from the theory of semigroups [3] that a semigroup $e^{t_{A}}$ is an inverse Laplace transform of the resolvent of the generator $A$, or more precisely, 


$$
e^{t A} u=s-\lim _{a \rightarrow \infty} \frac{1}{2 \pi i} \int_{\sigma-i a}^{\sigma+i a} e^{\lambda t}(\lambda-A)^{-1} u d \lambda
$$

holds for $u \in D(A)$ and $t \geq 0$ if $\sigma \in \mathbb{R}$ is large enough.

Write (3.1) for $B_{c}^{\infty}$ in $L^{2}\left(Q^{\infty}\right)$ and substitute in it the decomposition (I.6.11) of $\left(\lambda-B_{c}^{\infty}\right)^{-1}$ to deduce

$$
E_{c}^{\infty}(t)=\sum_{j=0}^{\bar{m}} \overleftarrow{U}_{j}(t, c)
$$

where $\stackrel{v}{U}_{j}=\mathcal{L}_{t}^{-1} U_{j}$ are given as follows. Let $\hat{B}_{c}(k)$ be that of (I.6.4), which also generates a semigroup in $L^{2}\left(\mathbb{R}_{\xi}^{n}\right)$, denoted as $\check{E}_{c}(t, k)$. Write (3.1) at this time for $\hat{B}_{c}(k)$. Then by (I.6.11),

$$
\overleftarrow{U}_{0}(t, c)=\mathscr{F}_{x}^{-1} \check{E}_{c}(t, k)\left\{\chi\left(|k|>\kappa_{0}\right)+\chi\left(|k|<\kappa_{0}\right) Q(k, c)\right\} \mathscr{F}_{x},
$$

where $Q(k, c)=\mathbb{I}-\Sigma \mathbb{P}_{j}(k, c)$, while. since $\mathcal{L}_{t}^{-1}(\lambda-\mu)^{-1}=e^{\mu t}$,

$$
\stackrel{\mathrm{U}}{j}_{j}(t, c)=\mathscr{F}_{x}^{-1} e^{\mu_{j}(k, c) t} \chi\left(|k|<\kappa_{0}\right) P_{j}(k, c) \mathscr{F}_{x}, \quad 1 \leq j \leq m .
$$

IProposition 3.2. Let $\sigma_{0}>0$ be the constant of (I.6.7). There exists a constant $\mathbb{C} \geq 0$ and

$$
\left\|\stackrel{v}{U}_{0}(t, c)\right\|_{\mathbb{B}\left(L^{2}\left(Q^{\infty}\right)\right)} \leq C e^{-\sigma_{0} t}, \quad t \geq 0
$$

Proof. Let $\hat{A}_{c}(k)$ be defined by (I.6.2). It generates a semigroup in $L^{2}\left(\mathbb{R}_{\xi}^{n}\right)$, denoted as $\mathscr{F}_{c}(t, k)$. By (3.1) for $\hat{B}_{c}(k)$ and $\hat{A}_{c}(k)$, and by the second resolvent equation of the form (I.6.14) for them,

$$
\begin{aligned}
& \stackrel{\vee}{E}_{c}(t, k)=\sum_{h=0}^{t-1} \mathbb{H}_{h}(t, k, c)+D_{l}(t, k, c) \\
& H_{h}=\left\{\left(\mathscr{F}_{c}(\circ, k) K_{c} *\right)^{h} \mathscr{F}_{c}(\circ, k)\right\}(t), \\
& D_{l}=\mathcal{L}_{t}^{-1} \hat{D}_{l}, \quad \hat{D}_{l}(\lambda, k, c)=\hat{G}^{l}\left(\lambda-\hat{B}_{c}(k)\right)^{-1}, \\
& \hat{G}=\left(\lambda-\hat{A}_{c}(k)\right)^{-1} K_{c} .
\end{aligned}
$$

Write $B=B\left(\mathbb{L}^{2}\left(\mathbb{R}_{\xi}^{n}\right)\right)$. Obviously $\mathscr{F}_{c}(t, k)=\left\{\exp \left(-i \xi \circ k-\nu_{c}(\xi)\right) t\right\} \times$, so in view of (I.3.4),

$$
\left\|\mathbb{H}_{h}(t, k, c)\right\|_{\mathbb{B}} \leq C t^{h} e^{-v_{0} t},
$$

uniformly for $k \in \mathbb{R}^{n}$. Next, combine Lemma $\mathbb{I} .6 .12$ (i) with (ii) restricted for $|k|>\kappa$, and choose $\kappa=(1+|\tau|)^{\gamma /(\gamma+1)}$ to obtain

$$
\|\hat{G}(\sigma+i \tau, k, c)\|_{\mathbb{B}} \leq C(1+|\tau|)^{-\gamma^{2} /(\gamma+1)},
$$

uniformly for $k \in \mathbb{R}^{n}$ and for $\sigma \geq-\nu_{0}+\delta, \delta>0$ being fixed. Then, by Proposition $\mathbb{I} .6 .3$ and (I.6.19) (i), $\hat{D}_{l}(\lambda, k, c)$ with the choice $l>\gamma^{-2}(\gamma+1)$ satisfies 
the condition of Lemma 2.3 of $g(\lambda)$ uniformly for $|k|>\kappa_{0}$ while so does $\hat{D}_{l}(\lambda, k, c) Q(k, c)$ uniformly for $|k|<\kappa_{0}$, both with $\sigma=-\sigma_{0}$. Hence

$$
\left.\begin{array}{l}
\sup _{|k|>\kappa_{0}}\left\|D_{l}(t, k, c)\right\|_{B}, \\
\sup _{|k|<\kappa_{0}}\left\|D_{l}(t, k, c) Q(k, c)\right\|_{B}
\end{array}\right\} \leq C e^{-\sigma_{0} t}
$$

Substitution of these estimates into (3.5) and then into (3.3) yields the proposition by the aid of Parseval.

Proposition 3.3. Under the same situation of Theorem 3.1

$$
\check{U}_{j}(t, c)\left(\mathbb{I}-P_{c}\right)^{m} \in \mathbb{B}_{\alpha}\left(\tilde{L}_{0}^{q, 2}, \tilde{L}_{\beta}^{p, \infty}\right), \quad 1 \leq j \leq \bar{m} \leq n+2 .
$$

Proof. Using (3.4) and proceeding exactly in the same way as in (I.6.12), we get

$$
\left\|\stackrel{v}{U}_{j}(t, c)\left(I-P_{c}\right)^{m} u\right\|_{\tilde{L}_{\beta}^{p, \infty}} \leq C I(t)\|u\|_{\tilde{L}_{0}^{q, 2}}
$$

with

$$
\mathbb{I}(t)=\left(\left.\left.\int_{|k|<k_{0}}\left|e^{\mu_{j}(k, c) t}\right| k\right|^{m}\right|^{r} d k\right)^{1 / r}, \quad \frac{1}{r}=\frac{1}{q}-\frac{1}{p} .
$$

Since $\operatorname{Re} \mu_{j}(k, c)=\operatorname{Re} \lambda_{j}(|k|)$ by Theorem I.6.2 (i), and by (I.6.6), $I(t) \leq$ $C(1+t)^{-\infty}$ for all $t \geq 0$, which proves the proposition.

To establish Theorem 3.1, we still need the following lemma on the semigroup $F_{c}^{\infty}(t)$ defined by (I.5.5). Put for $h \in \overline{\mathbb{N}}_{+}$,

$$
\begin{aligned}
& G_{h} *=\left(F_{c}^{\infty} K_{c} *\right)^{h}, \\
& H_{h}(t)=\left(G_{h} * F_{c}^{\infty}\right)(t) .
\end{aligned}
$$

Lemmm 3.4. (i) $\forall p \in[1, \infty], \forall \alpha \in \mathbb{R}, \forall \beta \in \mathbb{R}, \forall h \in \overline{\mathbb{N}}_{+}$,

$$
G_{h} * \in \mathbb{B}\left(\widetilde{L}_{\beta, \alpha}^{p, \infty}\right), \quad H_{h} \in \mathbb{B}\left(\widetilde{L}_{\beta}^{p, \infty}, \widetilde{L}_{\beta, \alpha}^{p \infty}\right) .
$$

(ii) $\forall p \in[2, \infty], \forall \beta \in \mathbb{R}, \exists l \in \overline{\mathbb{N}}_{+}, \forall \alpha \in \mathbb{R}_{\text {, }}$

$$
G_{l} * \in \mathbb{B}\left(\widetilde{L}_{0, \alpha}^{2,2}, \widetilde{L}_{\beta, \alpha}^{p, \infty}\right) \text {. }
$$

(iii) $\forall p \in[1, \infty], \forall \alpha \in \mathbb{R}, \forall \beta \in \mathbb{R}, \forall \delta \leq 1$,

$$
F_{c}^{\infty} \Lambda_{c}^{\delta} * \in \mathbb{B}\left(\tilde{L}_{\beta, a}^{p, \infty}\right) \text {. }
$$

Proof. Lemma I.5.1 implies that $F_{c}^{\infty}(t)$ satisfies the condition for $A(t)$ of Lemma 2.2 with $\delta=0$ and $\nu=\nu_{0}, X=\tilde{L}_{\beta}^{p, \infty}$. Then (i) follows readily by 
the aid of Proposition I.3.5. Similarly (ii) follows by a repeated use of Lemma I.5.1 (ii) (cf. the proof of Lemma I.5.4). And (iii) is a direct consequence of (2.2) for $\delta=0$ and $\nu=\nu_{c}(\xi)$. Note that the constant $C \geq 0$ of (2.2) is independent of $\nu$.

Proof of Theorem 3.1. Similarly to (3.5), we get

$$
E_{c}^{\infty}(t)=\sum_{h=0}^{l-1} H_{h}(t)+\left(G_{l} * E_{c}^{\infty}\right)(t),
$$

where $G_{l}$ and $H_{h}$ are of course those of (3.7). In view of Lemmas 3.4 (i) and (iii), it suffices to show that $G_{l} * E_{c}^{\infty}(t)$ enjoys Theorem 3.1. Choose $l \in N_{+}$of Lemma 3.4 (ii). Then Proposition 3.2 and Lemma 2.1 show that $G_{l} * \cup_{0}^{v}(\cdot, c) \in$ $\boldsymbol{B}\left(\tilde{L}_{0}^{2,2}, \tilde{L}_{\beta, \alpha}^{p, \infty}\right)$ for all $\alpha \geq 0$, while due to Proposition 3.3 and Lemma 3.4 (i), $G_{l} * \stackrel{v}{U}_{j}(\cdot, c), 1 \leq j \leq l, \in \mathbb{B}\left(\tilde{L}_{0}^{q, 2}, \tilde{L}_{\beta, \infty}^{p, \infty}\right)$ with the $\alpha$ specified in Theorem 3.1 . Now the proof is completed by (3.2).

Let $r^{ \pm}$be the trace operators given by Theorem 1.4.1. According to the theory of semigroups [3], $E_{c}^{\infty}(t) u \in D\left(B_{c}^{\infty}\right)$ whenever $u \in D\left(B_{c}^{\infty}\right)$. In virtue of Proposition I.4.4 and (I.5.1), therefore, $r^{ \pm} E_{c}^{\infty}(t) \in \boldsymbol{B}\left(W_{v_{c}}^{2,-}\left(Q^{\infty}\right), Y^{2, \pm}\right)$. We shall use this operator in $L^{\infty}$-space. Note that

$$
\left\|r^{ \pm} v\right\|_{\tilde{Y}_{\beta, \alpha}^{p, \infty, \pm}} \leq\|v\|_{L_{\beta+1 / p, \alpha}^{\infty}} .
$$

Combine this and Theorem 3.1 and put $p=\infty$ in both. Then we obtain

Proposition 3.5. Let $q \in[1.2]$ and $\beta \in \boldsymbol{R}$.

(i) Put $\alpha=\frac{n}{2 q}$. There is a constant $C \geq 0$ and

$$
\left\|\gamma^{ \pm} E_{c}^{\infty} u\right\|_{\tilde{Y}_{\beta, \alpha}^{\infty, \pm}} \leq C\left(\|u\|_{L_{\beta}^{\infty}}+\|u\|_{z^{q}}\right) .
$$

(ii) Let $m=0,1, \alpha \geq 0, \delta \leq 1$. Put $\alpha=\frac{1}{2}\left(\frac{n}{q}+m\right)$ and $\mu=\min (\alpha, \gamma, \alpha+$ $r-1)$. There is a constant $C \geq 0$ and if $r \neq 1$,

$$
\left\|\gamma^{ \pm} E_{c}^{\infty} *\left(I-P_{0}\right)^{m} \Lambda_{c}^{\delta} f\right\|_{Y_{\beta, \mu}^{\infty}, \pm} \leq C\left(\|f\|_{L_{\beta, \gamma}^{\infty}}+\left\|\Lambda_{c}^{\delta} f\right\|_{Z_{\gamma}^{q}}\right) .
$$

\section{§4. Asymptotic Behaviors of $\mathbb{E}_{c}(t)$}

$E_{c}(t)$ is the semigroup on the space $L^{2}(Q), Q=\Omega_{x} \times \mathbb{R}_{\xi}^{n}$, generated by the operator $B_{c}$ defined by (1.3) or more precisely by (I.7.10), and will also denote its realizations in other spaces. 
In this and following sections we use the spaces

$$
\begin{array}{ll}
\widetilde{L}_{\beta}^{p, q}=\widetilde{L}_{\beta}^{p, q}(Q), & \widetilde{L}_{\beta, \alpha}^{p, q}=\widetilde{L}_{\beta, \alpha}^{p, q}(Q), \\
X_{\beta}^{p}=\widetilde{L}_{\beta}^{\infty, \infty} \cap \tilde{L}_{\beta-1 / p}^{p, \infty}, & X_{\beta, \alpha}^{p}=\widetilde{L}_{\beta, \infty}^{\infty, \infty} \cap \widetilde{L}_{\beta-1 / p, \alpha}^{p, \infty}, \\
Z^{q}=\widetilde{L}_{0}^{2,2} \cap \widetilde{L}_{0}^{q, 2}, & Z_{\alpha}^{q}=\widetilde{L}_{0, \alpha}^{2,2} \cap \widetilde{L}_{0, \alpha}^{q, 2} .
\end{array}
$$

Let $\bar{c}_{0}>0$ be the constant of Proposition I.7.5 and write

$$
B\left[\bar{c}_{0}\right]=\left\{c \in \mathbb{R}^{n}|| c \mid \leq \bar{c}_{0}\right\} .
$$

In contrast to $E_{c}^{\infty}(t)$, asymptotic behaviors of $E_{c}(t)$ can be established only for $c \in B\left[\bar{c}_{0}\right]$.

Theorem 4. 1. Let $n \geq 3,1 \leq q \leq 2 \leq p \leq \infty, \beta>(n+1) / 2$ and $\theta \in[0,1)$. Put

$$
\alpha_{1}=\frac{1}{2}\left(\frac{1}{q}-\frac{1}{p}\right), \quad \alpha_{2}= \begin{cases}\frac{1}{2}(n-1+\theta), & (n, \text { odd }) \\ \frac{1}{2}(n-1), & (n, \text { even })\end{cases}
$$

(i) Put $\alpha=\min \left(\alpha_{1}, \alpha_{2}\right)$. There is a constant $C \geq 0$ and for all $c \in B\left[\bar{c}_{0}\right]$,

$$
\left\|E_{c} u\right\|_{X_{\beta, \alpha}^{p}} \leq C|c|^{-\theta}\left(\|u\|_{X_{\beta}^{p}}+\|u\|_{z^{q}}\right) \text {. }
$$

(ii) Put $\alpha=\min \left(\alpha_{1}+\frac{1}{2}, \alpha_{2}, \frac{1}{2}\left(1-\frac{1}{p}\right)\right)$. Let $\gamma \geq 0$ and put $\mu=\min (\alpha$, $\gamma, \alpha+\gamma-1)$. There is a constant $C \geq 0$, and for all, $c \in B\left[\bar{c}_{0}\right]$ and if $\gamma \neq 1$,

$$
\left\|E_{c} *\left(I-P_{0}\right) \Lambda_{c} f\right\|_{X_{\beta, \mu}^{p}} \leq C|c|^{-\theta}\left(\|f\|_{X_{\beta, \gamma}^{p}}+\left\|\Lambda_{c} f\right\|_{Z_{\gamma}^{q}}\right) .
$$

The proof is based on the expression of the resolvent of $B_{c}$ obtaintd in Theorem I.7.7, namely,

$$
\left(\lambda-B_{c}\right)^{-1}=r\left(\lambda-B_{c}^{\infty}\right)^{-1} e+S_{c}(\lambda) D_{c}(\lambda) M_{0}\left(\lambda-B_{c}^{\infty}\right)^{-1} e,
$$

where

$$
\begin{aligned}
& S_{c}(\lambda)=R_{c}(\lambda)+r\left(\lambda-B_{c}^{\infty}\right)^{-1} e K_{c} R_{c}(\lambda), \\
& D_{c}(\lambda)=\left(I-T_{c}(\lambda)\right)^{-1}, \\
& T_{c}(\lambda)=M_{0}\left(\lambda-B_{c}^{\infty}\right)^{-1} e K_{c} R_{c}(\lambda) .
\end{aligned}
$$

Suppose $\stackrel{\vee}{S}_{c}=\mathcal{L}_{t}^{-1} S_{c}$ and $\check{D}_{c}=\mathcal{L}_{t}^{-1} D_{c}$ exist. Then (4.1) implies, with the aid of (2.3), that

$$
E_{c}(t)=r E_{c}^{\infty}(t) e+\left(S_{c} * D_{c} * M_{0} E_{c}^{\infty} e\right)(t)
$$


First of all, we shall establish the existence of $\stackrel{\vee}{R_{c}}=\mathcal{L}_{t}^{-1} R_{c}$.

Proposition 4.2. $\stackrel{\vee}{R}_{c} *$ exists for all $c \in \mathbb{R}^{n}$ and has the following properties. Let $p, r \in[1, \infty], r \geq p$ and $\alpha, \beta \in \mathbb{R}$.

(i) $\stackrel{\mathrm{R}}{c}_{c} * \boldsymbol{B}\left(Y_{\beta, a}^{\infty,-}, \widetilde{L}_{\beta+\delta, \alpha}^{p, r}\right) \quad$ if $\delta>\frac{1}{p}+\frac{n}{r}$.

(ii) $K_{c} \check{R}_{c} * \in \mathbb{B}\left(Y_{\beta, \alpha}^{\infty,-}, \widetilde{L}_{\beta+\delta, \alpha}^{p, r}\right) \quad$ if $\delta>\frac{1}{p}+\frac{1}{r}-1$.

All the operator norms are locally bounded in $c$.

Proof. Let $h(t, x, \xi) \in \tilde{Y}_{\beta, a}^{p, r}-$ and define $v=v(t, x, \xi)$ by

$$
v=e^{-\nu_{c}(\xi) t^{-}(x, \xi)} h\left(t-t^{-}(x, \xi), x-t^{-}(x, \xi) \xi, \xi\right)
$$

for $(x, \xi) \in Q^{-}$and $t>t^{-}(x, \xi)$, and $v=0$ otherwise, where $Q^{-}$was defined in the course of the proof of Theorem I.4.1 and $t^{-}(X, \xi)$ in that of Proposition I.7.1. Put $w=|v|^{p}$ in (I.4.2)_ and use (I.7.3) to obtain

$$
\left.|i v(t, \cdot, \xi)|\right|_{L^{p}\left(\Omega_{x}\right)} ^{p}=\int_{0}^{t} \int_{\partial \Omega^{-}(\xi)}\left|e^{-v_{c}(\xi)(t-s)} h(s, X, \xi)\right|^{p} \times \rho(X, \xi) d \sigma_{x} d s,
$$

which, together with (I.3.4) and (2.2), gives

$$
\left\|e^{-\sigma t} v\right\|_{\tilde{L}_{\beta, \alpha}^{p, r}} \leq C\|h\|_{\tilde{Y}_{\beta, \alpha}^{p, r,-}},
$$

for all $\sigma>-\nu_{0}$ and $\alpha \in \mathbb{R}$. Hence $\hat{v}(\lambda)=\left(\mathcal{L}_{t} v\right)(\lambda)$ exists for $\lambda \in \mathbb{C}_{+}\left(-\nu_{0}\right)$. Compare (I.7.4) with (4.3) to see that $\hat{v}(\lambda)=R_{c}(\lambda) \hat{h}(\lambda)$, and recall (2.3). Thus (4.3) gives an explicit expression of $\stackrel{\vee}{r}_{c} *$ and (4.4) implies

$$
\stackrel{\vee}{R}_{c} * \in \mathbb{B}\left(\tilde{Y}_{\beta, \alpha}^{p, r,-}, \tilde{L}_{\beta, a}^{p, r}\right) \text {. }
$$

Now (i) follows since $Y_{\beta, \alpha}^{\infty,-} \subset \tilde{Y}_{\beta-\delta, \infty}^{p, r}$ is a continuous embedding, and then (ii) by Proposition I.3.5.

Remark 4.3. (4.3) indicates that $\mathscr{R}_{c}(t)$ itself exists only in the distribution sense in $t$.

By virtue of the above proposition, $\stackrel{\vee}{S}_{c} *$ exists and is given as

$$
\stackrel{v}{S}_{c} *=\stackrel{\vee}{R}_{c} *+r E_{c}^{\infty} e * K_{c} \stackrel{\vee}{R}_{c} *
$$

Lemma 4.4. Let $p \in[2, \infty], \beta>n / 2, \gamma \geq 0$ and $p u t$

$$
\alpha_{3}=\frac{n}{2}\left(1-\frac{1}{p}\right), \quad \mu=\min \left(\alpha_{3}, \gamma, \alpha_{3}+\gamma-1\right) \text {. }
$$

Then for all $c \in \mathbb{R}^{n}$, 


$$
\stackrel{\mathrm{S}}{\mathrm{S}}_{c}^{*} \in \mathbb{B}\left(T_{\beta-\overline{1} / p, \gamma}^{\infty,-}, \widetilde{L}_{\beta, \mu}^{p, \infty}\right),
$$

the norm being locally bounded in $c$.

Proof. In Theorem 3.1 (ii), put $q=1, m=\delta=0$ and substitute $e K_{c} \mathbb{R}_{c} * f$ for $f$. Use Proposition 4.2 (ii) thrice with $r=\infty$, with $p=r=2, \beta=0$ and with $p=1, r=2, \beta=0$. Then

$$
r E_{c}^{\infty} e * K_{c} \stackrel{v}{R}_{c} * \in \mathbb{B}\left(Y_{\beta, \gamma}^{\infty}, \overline{\mathcal{L}} \tilde{L}_{\beta, \mu}^{p, \infty}\right) .
$$

Combine this and Proposition 4.2 (i) in (4.5) to conclude the lemma.

As for $\check{D}_{c}(t)$, we have the

Proposition 4.5. $\check{D}_{c} *$ exists for all $c \in B\left[\bar{c}_{0}\right]$ and the following holds. Let $n \geq 3, \beta>n / 2, \gamma \geq 0$ and $\theta \in[0,1)$. Let $\alpha_{2}>0$ be as in Theorem 4.1 and put $\mu=\min \left(\alpha_{2}, \gamma, \alpha_{2}+\gamma-1\right)$. There is a constant $c \geq 0$ and for all $c \in B\left[\bar{c}_{0}\right]$ and if $\gamma \neq 1$,

$$
\left\|\check{D}_{c} *\right\|_{\mathbb{B}\left(Y_{\beta, \bar{\gamma}}^{\infty}, Y_{\beta, \mu}^{\infty}\right)} \leq C|c|^{-\theta}
$$

We relegate the lengthy proof of $\S 6$ and proceed to the

Proof of Theorem 4.1. In view of the assumption $[M]_{2}$ (iii) and Remark 1.1 of $\S \mathrm{I} .1, \tilde{M}_{0} E_{c}^{\infty}(t)$ also enjoys Proposition 3.5. Combine this with Lemma 4.4 and Proposition 4.5 and choose $r=\alpha_{2}$ in the last two. Add the results for $p=\infty$ and $p=p$, and note that $\alpha_{2} \geq 1$ for $n \geq 3$. It then follows that $\stackrel{\vee}{S}_{c} * \check{D}_{c} * \tilde{M}_{0} E_{c}^{\infty}(t)$ enjoys Theorem 4.1 (i) with $\alpha=\min \left(\alpha_{2}, \alpha_{3}, \frac{n}{2 q}\right)$ and Theorem 4.1 (ii) with $\alpha=\min \left(\alpha_{2}, \alpha_{3}, \frac{1}{2}\left(\frac{n}{q}+1\right)\right)$. This and Theorem 3.1, substituted in (4.2), complete the proof.

Finally we shall discuss the continuity in $t$ of $E_{c}(t)$.

Proposition 4.6. $E_{c}(t)$ is a strongly continuous semigroup on $\mathbb{L}^{p}(Q)$, $2 \leq p<\infty$.

Proof. The case $p=2$ was already proven. Therefore, the proposition follows if

$$
\left\|E_{c}(t)\right\|_{\mathbb{B}\left(L^{p}(Q)\right)} \leq C e^{\alpha t}
$$

holds for all $p \in[2, \infty]$. The constant $C \geq 0$ and $\alpha \in \mathbb{R}$ may depend on $p$. Indeed, let $u \in L^{\infty} \cap L^{2}$ and put $v=\left(E_{c}(t+h)-E_{c}(t)\right) u$, where $L^{p}=L^{p}(Q)$. Then by (4.6) for $p=\infty$, we get for each fixed $t$, 


$$
\|v\|_{L^{p}} \leq\|v\|_{L^{\infty}}^{1-2 / p}\|v\|_{L^{2}}^{2 / p} \leq C\|v\|_{L^{2}}^{2 / p}
$$

so that $v \rightarrow 0(h \rightarrow 0)$ in $L^{p}$ if $p \in[2, \infty)$. Again by (4.6) and since $L^{\infty} \cap L^{2}$ is dense in $L^{p}$, this results in the proposition. It remains to prove (4.6). Clearly this is the case for $p=2$, because $E_{c}(t)$ is strongly continuous, [3]. Let $A_{c}$ be the operator of $\S$ I.7. According to Theorem 7.6 (i), it generates a contraction semigroup in $L^{2}$, denoted as $F_{c}(t)$, and according to Theorem I.7.6 (iii),

$$
F_{c}(t)=r F_{c}^{\infty}(t) e+\left(\stackrel{v}{R}_{c} * \tilde{M}_{0} F_{c}^{\infty} e\right)(t)
$$

should hold. Evaluate this right side in $\mathbb{B}\left(L^{\infty}\right)$ using Lemma I.5.1 (i), (3.8), (4.3) and $[M]_{2}$ (iii). Then $F_{c}(t)$ enjoys (4.6) for $p=\infty$ and hence so does $E_{c}(t)$ as seen from the equation $E_{c}=F_{c}+F_{c} * K_{c} E_{c}$ and by Gronwall's inequality. Now the interpolation yields (4.6) for $p \in(2, \infty)$.

As a property of the convolution operator, we have

Corollary 4.7. $E_{c} *$ maps $L^{\infty}\left([0, \infty) ; L^{p}(Q)\right)$ into $\mathcal{E}^{0}\left([0, \infty) ; L^{p}(Q)\right), 2 \leq$ $p<\infty$.

\section{$\S 5 . \quad$ Stability of Stationary Solutions}

Define the nonlinear map $H$ by writing the right side of (1.4) as $H[w](t)$, that is,

$$
H[w]=E_{c} w_{0}+2 E_{c} * \Gamma_{0}[u(c), w(\cdot)]+E_{c} * \Gamma_{0}[w(\cdot), w(\cdot)] .
$$

We shall find fixed points of $H$. The spaces used in this section are all the same of the previous section.

In Theorem 1.9.3, we change the notations of the parameters $p, \theta$ and $\alpha$ to $p_{0}, \theta_{0}$ and $\alpha_{0}$ respectively, and fix them as well as $\beta$ in the region

$$
\begin{aligned}
& \theta_{0} \in[0,2 / 7), p_{0} \in[2.4] \cap\left(\left(1-\frac{2}{n+\theta_{0}}\right)^{-1}, n\right), \\
& \alpha_{0} \in\left(\theta_{0}\left(1+\frac{2}{p_{0}}\right), 1-\theta_{0}\left(2-\frac{1}{p_{0}}\right)\right), \beta>n+1 .
\end{aligned}
$$

Here the restriction on $p_{0}$ is somewhat stringent compared with (I.9.1) for a technical reason which will be revealed below.

In what follows, $\bar{c}=\bar{c}\left(\theta_{0}, p_{0}, \alpha_{0}, \beta\right)$ will denote the constant $\bar{c}$ of Theorem I.9.3 with values of the parameters specified by (5.1). Then the stationary solution $u=u(c)$ exists for each $c \in B[\bar{c}]$ with 


$$
\|u(c)\|_{X_{\beta}^{p_{0}}} \leq C|c|^{\alpha_{0}}
$$

The space in which the fixed point of $H$ is sought is $X_{\beta, \gamma}^{p}$ and the space from which the initials $w_{0}$ should be taken is $X_{\beta}^{p} \cap Z^{q}$. For $n \geq 4$, we can choose $p=p_{0}$ but not for $n=3$. More specifically, we should make the following choice.

$$
\begin{aligned}
& p \in[2,4] \cap\left(\left(1-\frac{2}{n}\right)^{-1},\left(\frac{1}{2}-\frac{1}{p_{0}}\right)^{-1}\right), \\
& q \in[1,2] \cap\left[1,\left(\frac{1}{p}+\frac{1}{n}\right)^{-1}\right), \\
& r \in\left(\frac{1}{2}, \gamma_{0}\right], r_{0}=\min \left(\frac{n}{2}\left(\frac{1}{q}-\frac{1}{p}\right), \frac{1}{2}\left(\frac{n}{p_{0}}+1\right), \frac{1}{2}\left(\frac{n}{p}+1\right)\right) . \\
& \beta>n+1 .
\end{aligned}
$$

Under this choice of parameters, we write

$$
\|\|\|=\|\left\|_{X_{\beta, \gamma}^{p},}\right\|\|=\|\left\|_{X_{\beta}^{p}}+\right\| \|_{z^{q}} .
$$

The complicated choice of parameters in the above is necessary in order for $H$ to be a contraction, which follows from the

Lemma 5.1. Let $n \geq 3$ and suppose (5.1) and (5.3) be fulfilled. Then for each $\alpha \in\left[0, \alpha_{0}\right)$, there are constants $C_{0}, C_{1} \geq 0$ such thai for all. $c \in B[\bar{c}]$,

$$
\begin{aligned}
& \left\|\left|H [ w ] \| | | \leq C _ { 0 } \| w _ { 0 } \left\|+C_{1}\left(|c|^{\alpha}+|\|w \mid\|)|\|w \mid\|,\right.\right.\right.\right. \\
& \left\|\left|H[w]-H\left[w^{\prime}\right]\right|\right\| \leq C_{1}\left(|c|^{\alpha}+\||w|\|+\left|\| w^{\prime}\right|||\right)\left|\left\|w-w^{\prime} \mid\right\| .\right.
\end{aligned}
$$

Proof. By Theorem 4.1 (i) with $\theta=0$,

$$
\left\|E_{c} w_{0}\right\| \mid \leq C_{0}\left\|w_{0}\right\|
$$

holds if $1 \leq q \leq 2 \leq p \leq \infty, 0 \leq r \leq \min \left(\alpha_{1}, \frac{1}{2}(n-1)\right)$. Next, put $f(t)=$ $\Lambda_{c}^{-1} \Gamma_{0}[u(c), w(t)] . \quad$ (I.9.3) readily gives

$$
\|f\|_{X_{\beta, \gamma}^{p}}+\left\|\Lambda_{c} f\right\|_{Z_{\gamma}^{q}} \leq C\|u(c)\|_{X_{\beta}^{p} p^{p}}\|w\|_{x_{\beta, \gamma}^{p}}
$$

if $\beta>n+1$ and $\frac{1}{p_{0}}+\frac{1}{p}=\frac{1}{q}$. With this $p, q$ and $f$ and with $\theta=\alpha_{0}-\alpha$, Theorem 4.1 (ii) yields

$$
\left\|| | E _ { c } * \Gamma _ { 0 } [ u ( c ) , w ] \left|\| \leq C _ { 1 } | c | ^ { \alpha - \alpha } 0 \| u ( c ) \left\|_{X_{\beta}^{p_{0}^{0}}}|\|w \mid\| .\right.\right.\right.
$$


Indeed, $\alpha_{1}+\frac{1}{2}=\frac{1}{2}\left(\frac{n}{p_{0}}+1\right)>1$ since $p_{0}<n$ due to (5.1), $\alpha_{2}>1$ since $\theta>0$ and $\frac{n}{2}\left(1-\frac{1}{p}\right)>1$ by (5.3), so that Theorem 4.1 (ii) applies with $\mu=$ $\min (\alpha, \gamma)=r$ for any $r$ subject to (5.3). And it is at this point that $p_{0}$ should satisfy the condition $p_{0}<n$ besides (I.9.1). Thus (5.1) is required. Finally, if $f(t)=\Lambda_{c}^{-1} \Gamma_{0}[v(t), w(t)]$, then (I.9.3) and (I.9.4) give

$$
\|f\|_{X_{\beta, 2 \gamma}^{p}}+\left\|\Lambda_{c} f\right\|_{Z_{2 \gamma}^{q}} \leq C\|\| v\|\|\|w\| \|
$$

Use again Theorem 4.1 (ii) but at this time with $q=p / 2, \theta=0$ and with $r$ replaced by $2 \gamma$. Since $p \in[2,4]$, then $q \in[1,2]$, and by (5.3),

$$
\alpha_{2}=\frac{1}{2}(n-1)>\alpha_{1}=\frac{1}{2}\left(\frac{n}{p}+1\right) \geq r_{0} \geq r>\frac{1}{2},
$$

so that $\mu=\min (\alpha, 2 \gamma) \geq \gamma$. In view of Lemma I.3.9 (i), therefore,

$$
\left\|\left|E _ { c } * \Gamma _ { 0 } [ v , w ] \left\||| \leq C_{1}|\|v\|\||\|w \mid\| \text {. }\right.\right.\right.
$$

Combine (5.2), (5.4), (5.6) and (5.8), and recall that $\Gamma_{0}$ is bilinear symmetric. This finishes the proof of the lemma.

Now we can prove the desired stability of $u(c)$ as a simple corollary to the

Theorem 5.2. Let $n \geq 3$. Under the assumptions $[\mathcal{O}],[q]$ and $[M]$ of $\S \mathbb{I} .1$, and for each $p, q, \beta, \gamma$ satisfying (5.3) and for $\alpha \in\left(0, \alpha_{0}\right)$, there exist positive constants $a_{0}$ and $\overline{\bar{c}}$ such that for any initial, $w_{0} \in X_{\beta}^{p} \cap Z^{q}$ with $\left\|w_{c}\right\| \leq a_{0}$, (1.4) possesses a unique solution

$$
w=w(t) \in X_{\beta, \gamma}^{p} \cap \mathcal{E}^{0}\left([0, \infty) ; L^{p}(Q)\right)
$$

in the large in time, if $c \in B[\bar{c}]$.

Proof. Let $C_{0}, C_{1}$ be the constants of Lemma 5.1 and choose constants $a_{0}$ and $\overline{\bar{c}}$ such that

$$
\begin{aligned}
& 0<\overline{\bar{c}} \leq \bar{c}, \quad \overline{\bar{c}}<C_{1}^{-1 / \alpha}, \\
& 0<a_{0}\left(1-C_{1} \overline{\bar{c}}^{\alpha}\right)^{2} /\left(4 C_{0} C_{1}\right)
\end{aligned}
$$

and put

$$
\mu=1\left\{\left(1-C_{1} \overline{\bar{c}}^{\alpha}\right)^{2}-4 C_{0} C_{1} a_{0}\right\}^{1 / 2}, \quad a_{1}=\left(\mu_{1}-C_{1} \bar{c}^{\alpha}\right) /\left(2 C_{1}\right) .
$$

Let $V$ be a ball 


$$
V=\left\{w(t) \in X_{\beta, \gamma}^{p}|||| w|| \mid \leq a_{1}\right\} .
$$

Endowed with the metric $d(v, w)=\|\| v-w \mid \|, V$ is a complete metric space. Suppose $\left\|w_{0}\right\| \leq a_{0}$ and $v, w \in V$. Then by the previous lemma,

$$
\begin{aligned}
& \|\| H[w] \| \mid \leq C_{0} a_{0}+\left(C_{1}\left(\bar{c}^{\alpha}+a_{1}\right) a_{1}=a_{1},\right. \\
& \|H[v]-H[w]\||| \leq C_{1}\left(\bar{c}^{\alpha}+2 a_{1}\right)|\|v-w\|=\mu|\|v-w\| .
\end{aligned}
$$

Since $\mu \in(0,1)$, this proves that $H: V \rightarrow V$ is a contraction. Hence a unique fixed point $w=H[w]$ exists in $V$ and gives a solution to (1.4) in the large in time. It is easy to check that this $w$ is a unique fixed point also in $X_{\beta, \gamma}^{p}$. It remains to show that

$$
w(t) \in \mathcal{E}^{0}\left([0, \infty) ; L^{p}(Q)\right)
$$

Note that $X_{\beta}^{p} \subset L^{p}(Q)$ is a continuous embedding if $\beta>n / p$. Hence $w_{0} \in L^{p}(Q)$, and in view of (5.5) and (5.7),

$$
\Gamma_{0}[u(c), w(t)], \quad \Gamma_{0}[w(t), w(t)] \in L^{\infty}\left([0, \infty) ; \mathbb{L}^{p}(Q)\right) .
$$

Then $H[w](t) \in \mathcal{E}^{0}\left([0, \infty) ; L^{p}(Q)\right)$ by Proposition 4.6 and its corollary. This completes the proof of the theorem.

Since $w \in V$, it follows that

$$
|w(t)|_{X_{\beta}^{p}} \leq a_{1}(1+t)^{-\gamma}
$$

whenever $\left\|w_{0}\right\| \leq a_{0}$. Thus $w(t) \rightarrow 0(t \rightarrow \infty)$ in $X_{\beta}^{p}$, which shows that the stationary solution $u=u(c)$ is asymptotically stable as $t \rightarrow \infty$.

\section{§6. Proof of Proposition 4.5}

Write $D_{c, l}(\lambda)=\left(\mathbb{I}-T_{c}(\lambda)\right)^{-1} T_{c}(\lambda)^{l}, l \in \mathbb{N}_{+}$, and note the expansion

$$
D_{c}(\lambda)=\sum_{h=0}^{l-1} T_{c}(\lambda)^{h}+D_{c, l}(\lambda)
$$

(see (4.1)). Referring to (2.3), we then get

$$
{\stackrel{\mathrm{D}}{D_{c}}}^{*}=\sum_{h=0}^{l-1}\left(\stackrel{\mathrm{v}}{T}_{c} *\right)^{h}+\stackrel{\mathrm{D}}{\mathrm{D}}_{c, l} *
$$

provided $\check{T}_{c}=\mathcal{L}_{t}^{-1} T_{c}$ and $\check{D}_{c, l}=\mathcal{L}_{t}^{-1} D_{c, l}$ exist. Apparently, $\check{T}_{c} *$ is given as

$$
\mathbb{T}_{c} *=\tilde{M}_{0} E_{c}^{\infty} e * K_{c} \stackrel{R}{R}_{c} *
$$

Lemma 6.1. $\forall c \in \mathbb{R}^{n}, \forall \beta>n / 2, \check{T}_{c} * \in \mathbb{B}\left(Y_{\beta, \gamma}^{\infty}, \bar{\gamma}\right)$ with $\gamma=n / 2$. Here the 
operator norm is locally bounded in $c$.

Proof. In Proposition 3.5 (ii), put $q=1, m=\delta=0$ and $r=n / 2$. Then $\alpha=\mu=r=n / 2$. Use thrice Proposition 4.2 (ii) as in the proof of Lemma 4.4. Then

$$
r^{ \pm} E_{c} e * K_{c} \check{R}_{c} * \in \boldsymbol{B}\left(Y_{\beta, \gamma}^{\infty},-, Y_{\beta, \bar{\gamma}}^{\infty}\right) .
$$

Hence the lemma follows by the assumption $[M]_{2}$ (iii) and Remark 1.1 of $\S$ I.1.

To prove the existence of $\stackrel{v}{D}_{c, l}(t)$, let $\Sigma\left(\bar{a}_{0}, \bar{\sigma}_{0}\right)$ be the closed domain of $\boldsymbol{C}$ given in Proposition I.7.5. Owing to Lemma I.7.3 (ii) and Proposition I.7.5 (iii), $D_{c, l}(\lambda)$ is analytic in $\lambda \in \Sigma\left(\bar{a}_{0}, \bar{\sigma}_{0}\right) \backslash\{0\}$, while, in view of Proposition I.7.4,

$$
\left\|D_{c, l}(\lambda)\right\| \leq C(1+|\lambda|)^{-l \gamma},
$$

both in $\boldsymbol{B}\left(Y^{2,-}\right)$. Choose $l>1 / r$. Then Lemma 2.3 can be applied to $X=$ $\boldsymbol{B}\left(Y^{2,-}\right)$ and $g(\lambda)=D_{c, l}(\lambda)$, so the integral

$$
\check{D}_{c, l}(t)=\frac{1}{2 \pi i} \int_{\sigma-i \infty}^{\sigma+i \infty} e^{\lambda t} D_{c, l}(\lambda) d \lambda, \quad \sigma>0,
$$

converges and $D_{c, l}=\mathcal{L}_{t} \stackrel{v}{D}_{c, l}$ holds, both in $\boldsymbol{B}\left(Y^{2,-}\right)$. Thus $\check{D}_{c, l}(t) \in \boldsymbol{B}\left(Y^{2,-}\right)$ exists for all $t \in \boldsymbol{R}$. But since Propositions I.7.4 and I.7.5 are valid also in $\boldsymbol{B}\left(Y_{\beta}^{\infty},-\right), \beta>n / 2$, then so is (6.2) with the same constant $\gamma>0$. Hence (6.3) converges also in $\boldsymbol{B}\left(Y_{\beta}^{\infty},-\right)$ and $\stackrel{\mathrm{D}}{\text { c.l }}(t) \in \boldsymbol{B}\left(Y_{\beta}^{\infty,-}\right)$.

To study asymptotic behaviors of $\stackrel{v}{D}_{c_{2} l}(t)$, we look again at Lemma I.7.3 and Proposition I.7.5 to note that

$$
D_{c, l}(\lambda) \in \mathscr{B}^{0}\left(\Sigma\left(\bar{a}_{0}, \bar{\sigma}_{0}\right) ; \boldsymbol{B}\left(Y^{2,-} \cap Y_{\beta}^{\infty,-}\right)\right) .
$$

Consequently it is possible by virtue of Cauchy's theorem to shift the path of integration in (6.3) to the boundary $\partial \Sigma\left(\bar{a}_{0}, \bar{\sigma}_{0}\right)$ of $\Sigma\left(\bar{a}_{1}, \bar{\sigma}_{0}\right)$, in the topology of $\boldsymbol{B}\left(Y^{2,-}\right)$. By (6.2), the integral on $\partial \Sigma\left(\bar{a}_{0}, \bar{\sigma}_{0}\right)$ converges in $\boldsymbol{B}\left(Y^{2,-}\right)$ as well as in $\boldsymbol{B}\left(Y_{\beta}^{\infty},-\right)$.

Put $\tau_{0}=\left(\bar{a}_{0} / \bar{\sigma}_{0}\right)^{1 / 2}$ and decompose $\partial \Sigma\left(\bar{a}_{0}, \bar{\sigma}_{0}\right)$ into two parts

$$
\begin{aligned}
& \Gamma^{(1)}=\left\{\lambda \in \boldsymbol{C}\left|\operatorname{Re} \lambda=-\bar{\sigma}_{0},\right| \operatorname{Im} \lambda \mid \geq \tau_{0}\right\}, \\
& \Gamma^{(2)}=\left\{\left.\lambda \in \boldsymbol{C}\left|\operatorname{Re} \lambda=-\bar{a}_{0}\right| \operatorname{Im} \lambda\right|^{2},|\operatorname{Im} \lambda| \leq \tau_{0}\right\},
\end{aligned}
$$

according to which $\stackrel{\mathrm{D}}{c, l}_{l}(t)$ is decomposed as

$$
\begin{aligned}
& \stackrel{\vee}{D}_{c, l}(t)=\check{\triangleright}^{(1)}(t)+\check{D}^{(2)}(t), \\
& \check{D}^{(j)}(t)=\frac{1}{2 \pi i} \int_{\Gamma(j)} e^{\lambda t} D_{c, l}(\lambda) d \lambda, \quad j=1,2 .
\end{aligned}
$$


In the rest of this section we write

$$
\mathbb{B}=\mathbb{B}\left(Y_{\boldsymbol{\beta}}^{\infty,-}\right)
$$

Lemma 6.2。 $\forall \beta>n / 2, \exists C>0 ; \forall t \geq 0, \forall c \in B\left[\bar{c}_{0}\right]$,

$$
\left\|\check{D}^{(1)}(t)\right\|_{B} \leq C e^{-\bar{\sigma}_{0} t} .
$$

Proof. Write $\lambda=\sigma+i \tau$. Then

$$
\check{D}^{(1)}(t)=\frac{1}{2 \pi} e^{-\bar{\sigma}_{0} t} \int_{|\tau| \geq \tau_{0}} e^{i \tau t} D_{c, l}\left(-\bar{\sigma}_{0}+i \tau\right) d \tau .
$$

In view of (6.2), the last integral converges and is uniformly bounded for $t \in \mathbb{R}$, in the topology of $\mathbb{B}$, whence the lemma follows.

Similarly we can get for $t \geq 0$,

$$
\left\|D^{(2)}(t)\right\|_{B} \leq C \int_{0}^{\tau_{0}} e^{-\bar{a}_{0} \tau t} d \tau \leq C(1+t)^{-1 / 2} .
$$

This can be improved as follows. Let $\alpha_{2}>0$ be that of Theorem 4.1.

Lemma 6.3. $\forall \theta \in[0,1), \forall \beta>n / 2, \exists C \geq 0 ; \forall t \geq 0, \forall c \in B\left[\bar{c}_{0}\right]$,

$$
\left\|D^{(2)}(t)\right\|_{B} \leq C|c|^{-\theta}(1+t)^{-a_{2}} \text {. }
$$

Before proving this lemma, we note that Lemmas 6.1, 6.2, 6.3 prove Proposition 4.4. Therefore, the remaining part of this section is devoted to the proof of Lemma 6.3.

Lemma 6.4. $\forall k \in \mathbb{N}_{+}, \forall c \in \mathbb{R}^{n}, \forall \beta \in \mathbb{R}, \exists C \geq 0 ; \forall \lambda \in \mathbb{C}_{+}\left(-\nu_{0}\right)$,

(i) $\left\|R_{c}^{(k)}(\lambda)\right\|_{\mathbb{B}\left(Y_{\beta}^{\infty},-, L_{\beta}^{\infty}\right)} \leq C\left(\operatorname{Re} \lambda+\nu_{0}\right)^{-k}$,

(ii) $\left\|K_{c} R_{c}^{(k)}(\lambda)\right\|_{\mathbb{B}\left(Y_{\beta}^{\infty,-}, L_{\beta-\gamma}^{p,-}\right)} \leq \mathbb{C}\left(\operatorname{Re} \lambda+\nu_{0}\right)^{-k-1 / p}, \gamma>\frac{1}{p}+\frac{1}{r}$.

Here $R_{c}^{(k)}(\lambda)=d^{k} R_{c}(\lambda) / d \lambda^{k}$, and the constant $C$ is locally bounded in $c_{\text {. }}$

Proof. Differentiate (I.7.4) with respect to $\lambda$. Then

$$
R_{c}^{(k)}(\lambda) h=\left(-t^{-}(x, \xi)\right)^{k} e^{-\left(\lambda+\nu_{c}(\xi)\right) t^{-}(x, \xi)} h\left(x-t^{-}(x, \xi) \xi, \xi\right)
$$

for $(x, \xi) \in Q^{-}$and $=0$ otherwise. Note that $\sup _{t \geqq 0} t^{k} e^{-t / 2}<\infty$ and proceed as in the proof of Lemma I.7.2 to conclude the lemma.

Let $\Sigma\left(a_{0}, \sigma_{0}\right)$ be that defined by (I.6.8).

Lemma 6.5. $\forall k \in \mathbb{N}_{+}, \forall c_{0}>0, \forall \beta \geq 0, \forall \theta \in[0,1), \exists C \geq 0 ; \forall \lambda \in \Sigma\left(a_{0}, \sigma_{0}\right)$, 
$\forall c \in B\left[c_{0}\right]$

$$
\left\|\left(\lambda-B_{c}^{\infty}\right)^{-k} u\right\|_{L_{\beta}^{\infty}} \leq C|c|^{-\theta}|\tau|^{\min (0, \gamma)}\left(\|u\|_{L_{\beta}^{\infty}}+\|u\|_{z^{1}}\right),
$$

where $r=n+\theta-2 k$.

Proof. Set, as in the proof of Theorem 1.6.7,

$$
\mathbb{R}=\left(\lambda-B_{c}^{\infty}\right)^{-1}, \quad R^{\prime}=\left(\lambda-A_{c}^{\infty}\right)^{-1}, \quad G=R^{\prime} K_{c} .
$$

As a property of the resolvent, we have

$$
d^{k} R / d \lambda^{k}=(-1)^{k} R^{k+1},
$$

and similarly for $R^{\prime}$. Hence differentiation of (I.6.14) yields

$$
R^{k}=A_{0}+\sum_{h=1}^{k}\left(\begin{array}{l}
k-1 \\
h-1
\end{array}\right) A_{k-h} R^{h},
$$

where

$$
\begin{aligned}
& A_{0}=(-1)^{k} \sum_{h=0}^{l-1} d^{k-1}\left(G^{h} R^{\prime}\right) / d \lambda^{k-1}, \\
& A_{h}=(-1)^{k-h} d^{h} G^{l} / d \lambda^{h} .
\end{aligned}
$$

In view of (6.5) for $R^{\prime}, A_{h}$ 's are expressed in linear combinations of products of $R^{\prime}$ and $K_{c}$. Therefore, similarly to Lemma I.5.4 and (I.6.15), we see that if $l>0$ is choosen large enough,

$$
A_{0} \in \mathbb{B}\left(L_{\beta}^{\infty}\right), \quad A_{h} \in \mathbb{B}\left(L_{\beta}^{\infty}\right) \cap \mathbb{B}\left(L^{2}, L_{\beta}^{\infty}\right)
$$

the norms being uniformly bounded for $(\lambda, c) \in \Sigma\left(a_{0}, \sigma_{0}\right) \times B\left[c_{0}\right]$. Recall that the decomposition (I.6.11) is orthogonal. Hence

$$
R^{k}=\sum_{j=0}^{\bar{m}} U_{j}(\lambda, c)^{k}
$$

holds. Substitute this into the last sum in (6.6) to deduce

$$
\left\|R^{k} u\right\|_{L_{\beta}^{\infty}} \leq C\left[\|u\|_{L_{\beta}^{\infty}}+\sum_{h=1}^{k}\left\{\left\|U_{0}(\lambda, c)^{h} u\right\|_{L^{2}}+\sum_{j=1}^{\bar{m}}\left\|U_{j}(\lambda, c)^{h} u\right\|_{L_{\beta}^{\infty}}\right\}\right] .
$$

Now the lemma readily follows from Propositions I.6.4 and I.6.6 for $p=\infty$, $q=1$.

Lemma 6.6. $\forall k \in \mathbb{N}_{+}, \forall \theta \in[0,1), \exists C \geq 0 ; \forall \lambda \in \Gamma^{(2)}, \forall c \in B\left[\bar{c}_{0}\right]$,

$$
\left\|T_{c}^{(k)}(\lambda)\right\|_{B} \leq C|c|^{-\theta}|\tau|^{\min (0, \gamma)}
$$

Here $T_{c}^{(k)}=d^{k} T_{c} / d \lambda^{k}$ and $r=n+\theta-2 k-2$.

Proof. In virtue of (6.5), 


$$
T_{c}^{(k)}=\sum_{h=0}^{k}(-1)^{h}\left(\begin{array}{c}
k \\
h
\end{array}\right) \tilde{M}_{0} R^{h+1} e K_{c} R_{c}^{(k-h)}(\lambda) 。
$$

Since $\tilde{M}_{0}$ satisfies $[M]_{2}(i i i)$, we have only to evaluate $\gamma^{ \pm} R^{h+1} e K_{c} R_{c}^{(k-h)}(\lambda)$. And this can be done by combining Lemmas 6.4 and 6.5 with (3.8) for $p=\infty$.

Lemma 6.7。 $D_{c, l}(\lambda)$ also enjoys the conclusion of Lemma 6.6.

Proof. For $D_{c}(\lambda)=\left(I-T_{c}(\lambda)\right)^{-1}$, we have

$$
d D_{c}(\lambda) / d \lambda=-D_{c}(\lambda)\left(d T_{c}(\lambda) / d \lambda\right) D_{c}(\lambda) .
$$

Compute $D_{c, l}^{(k)}(\lambda)=d^{k}\left(D_{c}(\lambda) T_{c}(\lambda)^{l}\right) / d \lambda^{k}$ by this and Leibniz' rule, and evaluate the resulting sum by Proposition I.7.5 and the above lemma to conclude the lemma.

Proof of Lemma 6.3. By integration by parts,

$$
2 \pi i \check{D}_{c, l}(t)=-\sum_{h=1}^{k}(-t)^{-h}\left[e^{\lambda t} D_{c, l}^{(h-1)}(\lambda)\right]_{\lambda=\bar{\lambda}_{0}^{\lambda}}^{\lambda=\lambda}+(-t)^{-h} \int_{\Gamma^{(2)}} e^{\lambda t} D_{c, l}^{(k)}(\lambda) d \lambda,
$$

where $\lambda_{0}=-\bar{\sigma}_{0}+i \tau_{0}$. Put $\lambda=\lambda_{0}, \bar{\lambda}_{0}$ in Lemma 6.7. Then the sum from $h=1$ to $k$ of $(6.7)$, denoted as $I_{1}(t)$, satisfies

$$
\left\|\left.\left|I_{1}(t)_{B} \| \leq C\right| c\right|^{-\theta} t^{-1} e^{-\bar{\sigma}_{0} t}, \quad t \geq 1 .\right.
$$

Denote the last integral in (6.7) as $I_{2}(t)$ and put $\mu=\min (0, n+\theta-2 k-2)$. By Lemma 6.7 it follows that if $\mu>-1$, then

$$
\left\|I_{2}(t)\right\|_{B} \leq C|c|^{-\theta} \int_{0}^{\tau_{0}} e^{-a_{0} \tau^{2} t} \tau^{\mu} d \tau \leq C|c|^{-\theta}(1+t)^{-(1+\mu) / 2} .
$$

We should choose $k$ as large as possible under the restriction $\mu>-1$. Therefore $k=(n-1) / 2$ when $n$ is odd and $k=(n-2) / 2$ when $n$ is even. Then $\alpha_{2}=k+(1+\mu) / 2$ is identical with that of Theorem 4.1, and Lemma 6.3 follows if (6.4) is taken into account.

\section{§7. Prow of Proposition 1.7 .4}

In what follows, $L_{\beta}^{p}$ will stand for either $L_{\beta}^{p}\left(Q^{\infty}\right)$ or $L_{\beta}^{p}(Q)$ with $\beta$ dropped when $\beta=0$. No confusions will arise. For each $c_{0}>0$ and $\delta>0$, put

$$
\begin{aligned}
\Sigma_{1} & =\overline{\mathbb{C}}_{+}\left(-\nu_{0}+\delta\right) \times B\left[c_{0}\right], \\
\Sigma_{2} & =\Sigma\left(a_{0}, \sigma_{0}\right) \times B\left[c_{0}\right],
\end{aligned}
$$

where $\Sigma\left(a_{0}, \sigma_{0}\right)$ was defined by (I.6.8). All the conclusions in the below are 
valid for arbitrarily fixed positive $c_{0}$ and $\delta$. Write $G=\left(\lambda-A_{c}^{\infty}\right)^{-1} K_{c}$ as before.

Lemma 7.耳. $\exists r>0 ; \forall \beta \geq 0, \forall p \in[2, \infty], \exists C \geq 0 ; \forall(\lambda, c) \in \Sigma_{1}$,

(i) $\|G\|_{B\left(L_{\beta}^{p}\right)} \leq C(1+|\lambda|)^{-\gamma / p}$,

(ii) $\left\|\tilde{M}_{0} G\right\|_{\mathcal{B}\left(L_{\beta}^{p}, Y_{\beta}^{p,-}\right)} \leq C(1+|\lambda|)^{-\gamma /(2 p)}$.

Proof. By Proposition 1.3.5 and Lemma I.5.2 (i),

$$
\|G\|_{\mathbb{B}\left(L_{\beta}^{p}\right)} \leq C\left(\operatorname{Re} \lambda+\nu_{0}\right)^{-1},
$$

while by (3.6) and Parseval,

$$
\|G\|_{B\left(L_{\beta}^{2}\right)} \leq C(1+|\operatorname{Im} \lambda|)^{-\gamma^{2} /(1+\gamma)},
$$

which proves (i) for $p=2$ if $\gamma^{2} /(1+\gamma)$ is rewritten as $r / 2$. Note that the proof of Lemma I.6.12, and subsequently that of (3.6), remain valid in $L_{\beta}^{2}$ if $\beta \neq 0$. Now (i) for $p>2$ follows by the interpolation between the result for $p=2$ and (7.1) for $p=\infty$. In order to prove (ii), we first repeat the proofs of (I.4.4) and (I.4.6)_ with $u$ replaced by $|u|^{2}$, noting that $\partial|u(X \pm t \xi, \xi)|^{2} / \partial t=$ $2 \operatorname{Re} \overline{u(X \pm t \xi, \xi}) \partial u(X \pm t \xi, \xi) / \partial t$. Then for $u \in W_{v_{c}}^{2, \pm}\left(Q^{\infty}\right)$,

$$
\left\|\gamma^{ \pm} u\right\|_{Y_{\beta}^{2, \pm}}^{2} \leq 2\|u\|_{L_{\beta}^{2}}\left\|\left(\lambda-A_{c}^{\infty}\right) u\right\|_{L_{\beta}^{2}} .
$$

Replace $u$ by $G u$ and substitute (i) to conclude

$$
\left\|\gamma^{ \pm} G\right\| \leq C(1+|\lambda|)^{-\gamma / 4} \quad \text { in } \quad \mathbb{B}\left(L_{\beta}^{2}, Y_{\beta}^{2, \pm}\right) .
$$

Then (ii) for $p=2$ is verified by $[M]_{2}$ (i). Note that

$$
\left\|r^{ \pm} u\right\|_{Y_{\beta}^{\infty}, \pm} \leq\|u\|_{L_{\beta}^{\infty}}
$$

Put $G u$ for $u$. Then $\tilde{M}_{0} G \in \mathbb{B}\left(L_{\beta}^{\infty}, Y_{\beta}^{\infty,-}\right)$ by (7.1) and $[M]_{2}$ (iii). Hence (ii) for $p>2$ follows again by the interpolation.

Proof of Proposition 1.7.4. (i). What we shall prove is that

$$
\left\|T_{c}(\lambda)\right\| \leq C(1+|\lambda|)^{-\gamma /(2 p)} \quad \text { in } \quad \mathbb{B}\left(Y_{\beta}^{p,-}\right)
$$

holds for all $(\lambda, c) \in \Sigma_{2}$. In view of Proposition 1.7.3, however, it is enough for this to hold for $|\lambda| \geq r_{0}$ with some $r_{0}>0$. Rewrite $T_{c}(\lambda)$ as

$$
T_{c}(\lambda)=\tilde{M}_{0} G S_{c}(\lambda),
$$

which follows with substitution of the second resolvent equation (I.6.14) for $l=1$ into the definition of $T_{c}(\lambda)$ (see (4.1)). Let $R=\left(\lambda-B_{c}^{\infty}\right)^{-1}$ and evaluate 
the right hand side of (I.6.14) with sufficiently large $l$ by the aid of Lemma I.5.4. Then

$$
\|R u\|_{L_{\beta}^{p}} \leq C\left(\|u\|_{L_{\beta}^{p}}+\|R u\|_{L^{2}}\right)
$$

for $p \geq 2$. By Proposition I.6.4, $\|R u\| \leq C\|u\|$ in $L^{2}$ if $|\lambda| \geq r_{0}$ with any $r_{0}>0$, the constant $C \geq 0$ being dependent on $r_{0}$ but not on $\lambda$. Consequently by Lemma I.7.2,

$$
\left\|S_{c}(\lambda) h\right\|_{L_{\beta}^{p}} \leq C\left(\|h\|_{Y_{\beta}^{p,-}}+\|h\|_{Y_{\gamma}^{p,-}}\right)
$$

for $p \geq 2, \beta \geq 0$ and $\gamma>\left(\frac{1}{2}-\frac{1}{p}\right)(n+1)-1$. Now Lemma 7.1 (ii) proves (7.3) for $(\lambda, c) \in \Sigma_{2},|\lambda| \geq r_{0}, r_{0}>0$ being arbitrary.

We proceed to the proof of Proposition I.7.4 (ii). Similarly to the above, it suffices to show that

$$
\left\|T_{c}(\lambda)^{3}\right\| \leq C(1+|\lambda|)^{-\gamma} \quad \text { in } \quad \mathbb{B}\left(Y_{\beta}^{\infty,-}\right)
$$

for $(\lambda, c) \in \Sigma_{2},|\lambda| \geq r_{0}$ with some $r_{0}>0$. To this end, we use

$$
T_{c}(\lambda)=\tilde{M}_{0} G e R_{c}(\lambda)+\tilde{M}_{0} G^{2} S_{c}(\lambda) \equiv T_{1}+T_{2},
$$

which is obtained by (I.6.14) for $l=2$.

Lemma 7.2. (i) $\forall p \in[2, \infty], \forall \beta \geq 0, \exists C \geq 0 ; \forall(\lambda, c) \in \Sigma_{1}$,

$$
\left\|T_{1}\right\| \leq C \quad \text { in } \mathbb{B}\left(Y_{\beta}^{p,-}, Y_{\beta+1}^{p,-1}\right) \text {. }
$$

(ii) $\forall p \in(n, \infty], \forall \beta>\left(\frac{1}{2}-\frac{1}{p}\right)(n+1)-1, \exists C \geq 0 ; \exists \delta>0 ; \forall(\lambda, c) \in \Sigma_{2}$,

$$
\left\|T_{2}\right\| \leq C \quad \text { in } \quad \mathbb{B}\left(Y_{\beta}^{p,-}, Y_{\beta+\bar{\delta}}^{\infty}\right) .
$$

Proof. (i) follows by Proposition 1.3.5, Lemmas I.5.5 and 1.7.2. By Lemma I.5.4 and (7.2),

$$
\left\|T_{2} h\right\|_{Y_{\beta}^{\infty,-}} \leq C\left\|S_{c}(\lambda) h\right\|_{Y_{\beta-\delta}^{p}},
$$

if $p>n$ and $\delta \leq 1-\frac{1}{p}$. Then (7.4) proves (ii).

From (7.6) it follows that

$$
T_{c}(\lambda)^{2}=T_{1}^{2}+T_{3}
$$

with $T_{3}=T_{1} T_{2}+T_{2} T_{1}+T_{2}^{2}$. By the above lemma, 


$$
\left\|T_{3}\right\| \leq C \quad \text { in } \quad \mathbb{B}\left(Y_{\beta-\delta}^{p,-}, Y_{\beta}^{\infty,-}\right)
$$

with $\delta \leq 2\left(1-\frac{1}{p}\right), p>n$. Let $p>n+1$ be fixed. In view of (I.7.6), (7.3) holds even if $\mathbb{B}\left(Y_{\beta}^{p,-}\right)$ is replaced by $\mathbb{B}\left(Y_{\beta}^{\infty,-}, Y_{\beta-\delta}^{p,-}\right)$ with $\delta>(n+1) / p$. Therefore $T_{3} T_{c}(\lambda)$ enjoys the same inequality of (7.5).

It remains to evaluate $T_{1}^{2}$, and it is at this stage that the type of boundary operator $M_{0}$ turns to be crucial and that the assumptions $[M]_{1}$ (iv) and $[M]_{2}$ (iv) are required. Estimates of different types are available as seen from the

Proposition 7.3。 Let $\beta \geq 0$.

(i) Suppose $M_{0}$ be given by (II.1.5) and fulfill $[M]_{1}$. Then for each $p \in$ $(n, \infty]$, there is a constant $C \geq 0$ such that for any $\varepsilon>0$ and $(\lambda, c) \in \Sigma_{1}$

$$
\left\|T_{1}^{2} h\right\|_{Y_{\beta}^{\infty,-}-} \leq C\left(\varepsilon\|h\|_{Y_{\beta-1}^{\infty,-1}}+\frac{1}{\varepsilon}\|h\|_{Y_{\beta-1}^{p,-}}\right) \text {. }
$$

(ii) Suppose $M_{0}$ fulfill $[M]_{2}$ and let $p_{0}$ be that of $[M]_{2}$ (iv). There is a constant $C \geq 0$ and for any $p \in\left[p_{0}, \infty\right]$ and $(\lambda, c) \in \Sigma_{1}$,

$$
\left\|T_{1}^{2}\right\| \leq C \quad \text { in } \quad \mathbb{B}\left(Y_{\beta-1}^{p,-}, Y_{\beta}^{\infty,-}\right) \text {. }
$$

Before proving this, we complete the

Proof of Proposition 1.7.4 (ii). As stated already, it suffices to show that $T_{1}^{2} T_{c}(\lambda)$ enjoys (7.5). Recall that (7.3) holds with $\mathbb{B}\left(Y_{\beta}^{p,-}\right)$ replaced by $\mathbb{B}\left(Y_{\beta}^{\infty},-\right.$, $\left.Y_{\beta-\delta}^{p,-}\right), \delta<(n+1) / p$. Choose again $p>n+1$. When $M_{0}$ is given by (I.1.5), put $T_{c}(\lambda) h$ for $h$ in Proposition 7.3 (i) and obtain

$$
\left\|T_{1}^{2} T_{c}(\lambda) h\right\|_{Y_{\beta}^{\infty,-}} \leq C\left(\varepsilon+\varepsilon^{-1}(1+|\lambda|)^{-\gamma /(2 p)}\right)\|h\|_{Y_{\beta}^{\infty,-}} .
$$

Then the desired estimate follows with the choice $\varepsilon=(1+|\lambda|)^{-\gamma /(4 p)}$. For the case of $[M]_{2}$, it suffices to combine Proposition 7.3 (ii) with (7.3).

The rest of this section is to be devoted to the proof of Proposition 7.3. Write $R^{\prime}=\left(\lambda-A_{c}^{\infty}\right)^{-1}$ and define

$$
V_{-}=R_{c}(\lambda) r^{-} R^{\prime}, \quad W_{-}=\tilde{M}_{c} R^{\prime} e K_{c} V_{-} e K_{c} R_{c}(\lambda) .
$$

Lemma 7。 $\forall p>n, \forall \beta \geq 0, V_{-} \in L^{\infty}\left(\Sigma_{1} ; \mathbb{B}\left(L_{\beta}^{p, \infty}, L_{\beta}^{\infty, p}\right)\right)$.

Proof. (I.5.5) and (I.7.4) yield an explicit expression

$$
\left(V_{-} u\right)(x, \xi)=\int_{t^{-}(x, \xi)}^{\infty} e^{-\left(\lambda+\nu_{c}(\xi)\right) t} u(x-t \xi, \xi) d t
$$


for $(x, \xi) \in Q^{-}$and $=0$ otherwise. Then the lemma can be proven exactly in the same way as Lemma $\mathbb{1}$.5.2 (ii).

In the above the space $L$ cannot be replaced by $\tilde{L}$.

Corollary 7.5. Under the same situation of Lemma 7.4,

$$
W_{-} \in L^{\infty}\left(\Sigma_{1} ; \mathbb{B}\left(Y_{\beta-\delta}^{p,-}, Y_{\beta}^{\infty,-}\right)\right), \delta<2\left(1-\frac{1}{p}\right)
$$

Proof. By Proposition I.3.5, $K_{c} \in L^{\infty}\left(\Sigma_{1} ; \mathbb{B}\left(L_{\beta-\delta}^{p}, L_{\beta}^{p, \infty}\right)\right)$ if $p>n / 2, \delta<$ $1-1 / p$, so by Lemma $\mathbb{I} .7 .2$ (i), $K_{c} \mathbb{R}_{c}(\lambda) \in L^{\infty}\left(\Sigma_{1} ; \mathbb{B}\left(Y_{\beta}^{p,-}, L_{\beta}^{p, \infty}\right)\right)$. Similarly, $\tilde{M}_{0} R^{\prime} e K_{c} \in L^{\infty}\left(\Sigma_{1} ; \mathbb{B}\left(L_{\beta}^{\infty}, p, Y_{\beta+\delta}^{\infty}-\right)\right)$ by the aid of $\mathbb{L e m m a} \mathbb{I} .5 .5$ (i) and $[M]_{2}$ (iii). Combine these with Lemma 7.4.

We should also define

$$
V_{+}=R_{c}(\lambda) M_{0} \gamma^{+} R^{\prime}, \quad W_{+}=\tilde{M}_{0} R^{\prime} e K_{c} V_{+} e K_{c} R_{c}(\lambda)
$$

Apparently $T_{1}^{2}=W_{+}-W_{-}$.

Proof of Proposition 7.3 (ii). We have only to show that $W_{+}$enjoys the conclusion of Corollary 7.5. Similarly to (3.8), $\gamma^{+} \in \mathbb{B}\left(L_{\beta}^{\infty, p}, Y_{\beta}^{\infty, p,+}\right)$. Then by Lemma I.5.2 (ii) with $p=\infty, \gamma^{+} R^{\prime} \in L^{\infty}\left(\Sigma_{1} ; \mathbb{B}\left(L_{\beta}^{p, \infty}, L_{\beta}^{\infty, p,+}\right)\right)$ if $p>n$. Now we shall use $[M]_{2}$ (iv), in which it may be assumed that $p_{0}>n$ (if not, take an interpolation with $[M]_{2}$ (iii)). Then $M_{0} r^{+} R^{\prime} \in L^{\infty}\left(\Sigma_{1} ; \mathbb{B}\left(L_{\beta}^{p_{0}, \infty}, Y_{\beta}^{\infty},-\right)\right)$, and by Lemma I.7.2 (i), $V_{+} \in L^{\infty}\left(\Sigma_{1} ; \mathbb{B}\left(L_{\beta}^{p_{0}, \infty}, L_{\beta}^{\infty}\right)\right)$. A slight modification of the proof of Corollary 7.5 then leads to the desired result.

The proof of Proposition 7.3 (i) is far more complicated. Since $M_{0}$ is given by (I.1.5) and by virtue of (I.5.5) (ii) and (I.7.4), $V_{+}$can be written as

$$
V_{+} u=\int_{0}^{\infty} e^{-\psi(t)} u(X-t \tilde{m}, \tilde{m}) d t
$$

for $(x, \xi) \in Q^{-}$and $=0$ for $(x, \xi) \in Q \backslash Q^{-}$, where

$$
\begin{aligned}
& \psi(t)=\left(\lambda+\nu_{c}(\xi)\right) t^{-}(x, \xi)+\left(\lambda+\nu_{c}(\tilde{m})\right) t \\
& X=X(x, \xi)=x-t^{-}(x, \xi) \xi \\
& \widetilde{m}=\widetilde{m}(x, \xi)=m(X(x, \xi), \xi)
\end{aligned}
$$

$m(x, \xi)$ being subject to $[M]_{1}$. Write $\tilde{\xi}=\xi /|\xi|$. The following homogeneity properties come from $[M]_{1}$ (iv) and (I.7.3) (iv). 


$$
\begin{aligned}
& t^{-}(x, \xi)=t^{-}(x, \tilde{\xi}) /|\xi|, \\
& X(x, \xi)=X(x, \tilde{\xi}), \\
& \tilde{m}(x, \xi)=|\xi| \tilde{m}(x, \tilde{\xi}),
\end{aligned}
$$

all for $(x, \xi) \in Q^{-}$. Put

$$
\Xi(x)=\left\{\xi \in \boldsymbol{R}^{n} \mid(x, \xi) \in Q^{-}\right\},
$$

and define the map $y=y(\xi)$ by

$$
\Xi(x) \ni \xi \rightarrow y=X(x, \xi)-\widetilde{m}(x, \xi) \in \boldsymbol{R}^{n},
$$

where $x \in \Omega$ is arbitrarily fixed. Define for each $t_{0}, b, \delta>0$,

$$
\Xi_{1}(x)=\left\{\xi \in \Xi(x)\left|t^{-}(x, \tilde{\xi}) \leq t_{0}, \delta \leq\right| \xi \mid \leq b, \rho(X(x, \tilde{\xi}), \tilde{\xi}) \geq \delta\right\} .
$$

Let $y_{\xi}$ denote Jacobi's matrix $\left(\partial y_{j} / \partial \xi_{k}\right)$.

Lemma 7.6. $\forall \delta>0, \exists t_{0}>0 ; \exists m_{0}>0 ; \forall b>0, \forall x \in \Omega, \forall \xi \in \Xi_{1}(x)$,

$$
\left|\operatorname{det} y_{\xi}\right| \geq m_{0} \text {. }
$$

Proof. The assumption [O] of $\S \mathrm{I}$.1 implies that $\partial \Omega$ is represented locally by the equation $\phi(x)=0, \phi$ being a piecewise smooth function. Then $t=$ $t^{-}(x, \xi)$ is a unique solution to $\phi(x-t \xi)=0$. Differentiate this and deduce

$$
\nabla_{\xi} t^{-}(x, \xi)=t^{-}(x, \xi) \rho(X, \xi)^{-1} n(X),
$$

where $n(X)$ is the unit outward normal to $\partial \Omega$ and $\rho(X, \xi)=|n(X) \cdot \xi|$ at $X=X(x, \tilde{\xi})$. Consequently Jacobi's matrix $X_{\xi}$ is given as

$$
\begin{aligned}
& X_{\xi}(x, \xi)=-t^{-}(x, \xi) B(X(x, \tilde{\xi}), \tilde{\xi}), \\
& B(X, \tilde{\xi})=I_{n}+\rho(X, \tilde{\xi})^{-1}\left(\tilde{\xi}_{j} n_{k}(X)\right),
\end{aligned}
$$

$I_{n}$ being a unit matrix of order $n$. The $n \times n$ matrix $B(X, \xi)$ has, in view of $[\Theta]$ and $[M]_{1}(\mathrm{i})$, piecewise smooth and uniformly bounded entries as far as $\rho(X, \tilde{\xi}) \geq \delta>0$. By (7.8) and (7.9),

$$
\tilde{m}_{\xi}(x, \xi)=m_{X} X_{\xi}+m_{\xi}=-t^{-}(x, \tilde{\xi}) m_{X}(X, \tilde{\xi}) B(X, \tilde{\xi})+m_{\xi}(X, \tilde{\xi})
$$

is homogeneous of degree 0 with piecewise smooth and uniformly bounded entries if $\rho(X, \tilde{\xi}) \geq \delta, t^{-}(X, \tilde{\xi}) \leq t_{0}$. Then

$$
y_{\xi}=X_{\xi}-\tilde{m}_{\xi}=-t^{-}(x, \xi)\left(\frac{1}{|\xi|} I_{n}-m_{x}(X, \tilde{\xi})\right) \times B(X, \tilde{\xi})-m_{\xi}(X, \tilde{\xi}) .
$$

Replace $t^{-}(x, \tilde{\xi)}$ by $t$ in the last expression and denote its determinant by 
$J(t)=J(t, X, \tilde{\xi},|\xi|)$. Then $J(t)$ is a polynomial of $t$ of order at most $n$, whose coefficients are all piecewise smooth and uniformly bounded if $\rho(X, \tilde{\xi}) \geq \delta$ and $|\xi| \geq \delta$. Therefore $J(t) \rightarrow J(0)$ as $t \rightarrow 0$ uniformly, and

$$
|J(0)|=\left|\operatorname{det} m_{\xi}(X, \tilde{\xi})\right| \geq m_{0}
$$

by $[M]_{1}$ (iii), so $|J(t)| \geq m_{0} / 2$ for $0 \leq t \leq t_{0}$ with some $t_{0}>0$. Since $\operatorname{det} y_{\xi}=$ $J\left(t^{-}(x, \tilde{\xi})\right)$, this proves the lemma.

Next, define $\Xi_{2}(x)=\Xi_{2}\left(x, t_{1}, b, \delta\right)$ by

$$
\Xi_{2}(x)=\left\{\xi \in \Xi(x)\left|t^{-}(x, \tilde{\xi}) \geq t_{1},\right| \xi \mid \leq b, \rho(X(x, \tilde{\xi}), \tilde{\xi}) \geq \delta\right\} .
$$

Lemma 7.7. $\exists m_{0}>0 ; \forall \delta>0, \forall x \in \Omega, \forall t_{1}>0, \forall b>0, \exists \Xi_{2}^{\prime}(x) \subset \Xi_{2}(x)$;

(i) $\operatorname{mes} \Xi_{2}^{\prime}(x) \leq m_{0} b^{n-1} \delta$,

(ii) $\left|\operatorname{det} y_{\xi}\right| \geq m_{0}\left(t_{1}-\delta / b\right)^{n-1}, \xi \in \Xi_{2}(x) \backslash \Xi_{2}^{\prime}(x)$.

Proof. Rewrite (7.11) as

$$
y_{\xi}=-t^{-}(x, \xi) B(X, \tilde{\xi})-\tilde{m}_{\xi}(x, \xi) .
$$

For each fixed $X \in \partial \Omega$, we may choose the coordinates such that $n(X)=$ $(0,0, \cdots, 0,1)$. Then for $(X, \xi) \in S^{-}$(see $\left.\S \mathbb{I} .4\right)$,

$$
\rho(X, \xi)=-\xi_{n} .
$$

Let $\omega^{\prime}=-\tilde{\xi}_{n}^{-1 t}\left(\tilde{\xi}_{1}, \tilde{\xi}_{2}, \cdots, \tilde{\xi}_{n-1}\right)$ be a column vector of order $n-1$. Then in our choice of coordinates,

$$
B(X, \xi)=\left(\begin{array}{cc}
I_{n-1} & \omega^{\prime} \\
0 & 0
\end{array}\right)
$$

Write $\tilde{m}_{\xi}=\left(\widetilde{m}_{j k}\right), 1 \leq j, k \leq n$, and put $m^{1}=\left(\tilde{m}_{j, k}\right), 1 \leq j, k \leq n-1$, (a matrix of order $n-1), m^{2}={ }^{t}\left(m_{1 n}, m_{2 n}, \cdots, m_{n-1 n}\right)$ (a column vector of order $\left.n-1\right), m^{3}=$ $\left(\tilde{m}_{n 1}, \tilde{m}_{n 2}, \cdots, \tilde{m}_{n n-1}\right)$ (a law vector of order $n-1$ ) and $m^{4}=\tilde{m}_{n n}$. Then in the same coordinates,

$$
\begin{aligned}
& \operatorname{det} y_{\xi}=\left(-t^{-}(x, \xi)\right)^{n-1} J_{0}(x, \tilde{\xi},|\xi|), \\
& J_{0}(x, \xi, t)=\left|\begin{array}{cc}
I_{n-1}+t \mu m^{1} & \omega^{\prime}+t \mu m^{2} \\
m m^{3} & m^{4}
\end{array}\right|
\end{aligned}
$$

where $\mu=t^{-}(x, \tilde{\xi})^{-1}$. Obviously $J_{0}$ is a polynomial of $t$ of order at most $n-1$, 
and its coefficients are all piecewise smooth and uniformly bounded for $x \in \Omega$, $\xi \in \Xi_{2}(x)$. Noting (7.13), we see

$$
J_{0}(x, \tilde{\xi}, 0)=m^{4}-m^{3} \cdot \omega^{\prime}=\tilde{\xi}_{n}^{-1}\left(\tilde{\xi} \circ \nabla_{\xi} m_{n}\right)(X, \tilde{\xi}) .
$$

By (7.8), $\tilde{\xi} \cdot \nabla_{\xi} m_{n}=\partial m_{n}(X,|\xi| \tilde{\xi}) / \partial|\xi|=m_{n}(X, \tilde{\xi})$, and in our choice of coordinates, $\tilde{\xi}_{n}^{-1} m_{n}(X, \tilde{\xi})=-\rho(X, \tilde{\xi})^{-1} \rho(X, \tilde{m})$. Hence by virtue of $[M]_{1}$ (i) (iii), there holds with some constant $m_{0}>0$,

$$
\left|J_{0}(x, \tilde{\xi}, 0)\right|=\left|\operatorname{det} m_{\xi}(X(x, \tilde{\xi}), \tilde{\xi})\right|^{-1} \geq m_{0},
$$

for all $(x, \tilde{\xi}) \in Q^{-}$. Obviously this result is free from the choice of coordinates. As a consequence, the polynomial $J_{0}$ of $t$ does not vanish identically, and thereby it has at most $n-1$ distinct real zeros $t=t_{j}(x, \tilde{\xi}), 1 \leq j \leq j_{0}$, where the number $j_{0}$ of the zeros may of course vary with $x$ and $\tilde{\xi}$. Let us show that

$$
\Xi_{2}^{\prime}(x)=\left\{\xi \in \Xi_{2}(x)|||\xi|-t_{j}(x, \xi) \mid \leq \delta, 1 \leq j \leq j_{0}\right\}
$$

is the desired subset stated in the lemma. Since $t_{j}$ 's are independent of $|\xi|$, (i) of the lemma is evident. Since $t_{j} \neq 0$ by (7.15), we can write

$$
J_{0}(x, \tilde{\xi},|\xi|)=J_{0}(x, \tilde{\xi}, 0) \prod_{j=1}^{j_{0}}\left(1-\frac{|\xi|}{t_{j}(x, \xi)}\right)^{k_{j}}
$$

$k_{j}$ being the multiplicity of $t_{j}$. Let $\xi \in \Xi_{2}(x) \backslash \Xi_{2}^{\prime}(x)$. It $\left|t_{j}\right| \leq 2 b$, then

$$
\left|1-\frac{|\xi|}{t_{j}}\right| \geq|| \xi\left|-t_{j}\right| /\left|t_{j}\right| \geq \delta /(2 b),
$$

while if $\left|t_{j}\right| \geq 2 b$, then

$$
\left|1-\frac{|\xi|}{t_{j}}\right| \geq 1-|\xi| /(2 b) \geq 1 / 2 .
$$

This, together with (7.15) and (7.16), gives $\left|J_{0}\right| \geq m_{0}(\delta /(2 b))^{n-1}$, whence (ii) of the lemma follows in virtue of (7.14).

Proposition 7.8. $\forall p>n, \forall \beta \geq 0, \exists C \geq 0 ; \forall a>0, \forall \varepsilon>0$,

$$
\left\|\chi(|\xi|<a) V_{+} u\right\|_{L_{\beta}^{\infty, p}} \leq C a^{n}\left(\varepsilon\|u\|_{L_{\beta}^{\infty}}+\varepsilon^{-1}\|u\|_{L_{\beta}^{p, \infty}}\right) .
$$

Proof. Recall (7.7), and put $v(x)=\|u(x, \cdot)\|_{L^{\infty}\left(\mathbb{R}_{\xi}^{n}\right)}$ and

$$
w(x, t, a)=\|X(|\xi|<a) v(\chi(x, \xi)-t \tilde{m}(x, \xi))\|_{L^{p}\left(\boldsymbol{R}_{\xi}^{n}\right)} .
$$

The proof of the proposition will be carried out only for $\beta=0$ because the 
case $\beta \neq 0$ can be dealt with exactly in the same way. Let $(\lambda, c) \in \Sigma_{1}$, but in the below we change the notation $\delta$ by $\delta_{0}$ in the definition of $\Sigma_{1}$. Since $\operatorname{Re} \psi(t) \geq \delta_{0} t,(7.7)$ leads to

$$
\left.\| \chi(|\xi|<a) V_{+} u\right) \|_{L^{p}\left(\mathbb{R}_{\xi}^{n}\right)} \leq \int_{0}^{\infty} e^{-\delta_{0} t}{ }^{\prime}(x, t, a) d t .
$$

Denote the last integral as $I$. Given an $\varepsilon>0$, put $a^{\prime}=|\log \varepsilon| / \delta_{0}$ and split the integral $I$ as

$$
I=\int_{a^{\prime}}^{\infty}+\int_{0}^{a^{\prime}} \equiv I_{1}+I_{2}
$$

Apparently $\sup _{t \geq 0} w(x, t, a) \leq C a^{n / p}\|u\|_{L^{\infty}}$, independently of $x$, so

$$
I_{1} \leq C \varepsilon a^{n / p}\|u\|_{L^{\infty}},
$$

while since $w(x, t, a) \leq t^{-n / p} w(x, 1, a t)$ by (7.8) and by the change of variables $t \xi \rightarrow \xi$, and since $w$ is monotone increasing in $a$, then

$$
I_{2} \leq C w\left(x, 1, a a^{\prime}\right)
$$

holds for $p>n$. In Lemmas 7.6 and 7.7, put

$$
\delta=\varepsilon^{p}, \quad b=a a^{\prime}=a|\log \varepsilon| / \delta_{0}, \quad t_{1}=t_{0},
$$

where $t_{0}$ is the constant of Lemma 7.6. Define

$$
\Xi_{3}(x)=\{\xi \in \Xi(x) \cap B[b]|| \xi \mid \leq \delta \text { or } \rho(X(x, \tilde{\xi}), \tilde{\xi}) \leq \delta\} .
$$

Then $B[b]=\left\{\xi \in \mathbb{R}^{n}|| \xi \mid \leq b\right\}$ can be expressed as the union of $\Xi_{j}(x), j=1,2,3$ and recalling the map $y=y(\xi)$,

$$
w(x, 1, b)^{p}=\int_{B[b]}|v(y(\xi))|^{p} d \xi=\sum_{j=1}^{3} \int_{z_{j}(x)} \equiv \sum_{j=1}^{3} I_{2 j} .
$$

By Lemma 7.6,

$$
I_{21}=\int_{\Xi_{1}(x)}|v(y)|^{p}\left|\operatorname{det} y_{\xi}\right|^{-1} d y \leq C \int_{\mathbb{R}^{n}}|v(y)|^{p} d y=C\|u\|_{L^{p, \infty}}^{p},
$$

while by Lemma 7.7,

$$
\begin{gathered}
I_{22}=\int_{\Xi_{2}^{\prime}(x)}+\int_{\Xi_{2}(x) \backslash \Xi_{2}^{\prime}(x)} \\
\quad \leq\left(\operatorname{mes} \Xi_{2}^{\prime}(x)\right)\|v\|_{L^{\infty}}^{p}+C\left(\frac{b}{\delta t_{0}}\right)^{n-1}\|v\|_{L^{p}}^{p} \\
\quad \leq C\left(\varepsilon^{p+1} \mid\left\|_{1} u\right\|_{L^{\infty}}^{p}+\left(a \varepsilon^{-p}\right)^{n-1}\|u\|_{L}^{p} p\right) .
\end{gathered}
$$


Finally, it is easily checked that mes $\Xi_{3}(x) \leq C\left(\delta^{n}+b \delta^{n-1}\right)$ holds uniformly for $x \in \Omega$, so that

$$
I_{23} \leq C a \varepsilon^{p n}\|u\|_{L^{\infty}}^{p} .
$$

Combining all the estimates so far obtained completes the proof of the proposition.

Proof of Proposition 7.3 (i). Owing to Corollary 7.5, it suffices to show that $W_{+}$enjoys Proposition 7.3 (i). Use Proposition I.3.5 twice. Then the above proposition yields

$$
\| K_{c} \chi\left(|\xi|<a ; V_{+} K_{c} u \|_{L_{\beta}^{\infty}} \leq C a^{n}\left(\varepsilon\|u\|_{L_{\beta-2}^{\infty}}+\frac{1}{\varepsilon}\|u\|_{L_{\beta-\gamma}^{p}}\right)\right.
$$

for $r<2\left(1-\frac{1}{p}\right)$, while since $\left\|V_{+}\right\| \leq C$ in $\boldsymbol{B}\left(L_{\beta}^{\infty}\right)$ as seen from $[M]_{2}$ (iii),

Remark I.1.1 and Lemmas I.5.5 and I.7.2, then by the use of (I.3.7),

$$
\left\|K_{c} \chi(|\xi|>a) V_{+} K_{c} u\right\|_{L_{\beta}^{\infty}} \leq C(1+a)^{-1}\|u\|_{L_{\beta-1}^{\infty}} .
$$

Choose $a=\varepsilon^{-1 /(n+1)}$ and replace $\varepsilon^{1 /(n+1)}$ by $\varepsilon$. Then

$$
\left\|K_{c} V_{+} K_{c} u\right\|_{L_{\beta}^{\infty}} \leq C\left(\varepsilon\|u\|_{L_{\beta-1}^{\infty}}+\frac{1}{\varepsilon}\|u\|_{L_{\beta-1}^{p}}\right) \text {. }
$$

Now the desired estimate for $W_{+}$follows readily from Lemmas I.5.5 and I.7.2.

\section{References}

[1] Asano, K., On the initial boundary value problem of the nonlinear Boltzmann equation in an exterior domain, to appear.

[2 2 Caflisch, R.E., The Blotzmann equation with a soft potential, I and II, Comm. Math. Phys., 74, (1980) 71-95 and 97-109.

[ 3 ] Hille, E. and Phillips, R.S., "Functional Analysis and Semigroups", Revised Ed, Providence, Amer. Math. Soc. Colloq. Publ., Vol. 31. 1957.

[4] Nishida, T. and Imai, K., Global solutions to the initial value problem for the nonlinear Boltzmann equation, Publ. R.I.M.S., Kyoto Univ., 12, (1976) 229-239.

[ 5 ] Shizuta, Y. and Asano, K.. Global solutions of the Boltzmann equation in a bounded domain, Proc. Japan Acad., 53, Ser. A, (1977) 3-5.

[6] Ukai, S., On the existence of global solutions of a mixed problem for the nonlinear Boltzmann equation, Proc. Japan Acad., 50, (1974) 179-184.

[ 7 ] — L L L solutions globales de l'équation de Boltzmann dans l'espace tout entier et dans le demi-espace, C. R. Acad. Sci., Paris, 282A, (1976) 317-320.

[8] Ukai, S. and Asano, K., On the Cauchy problem of the Boltzmann equation with soft potentials, Publ. R.I.M.S. Kyoto Univ., 18, (1982) 477-519.

[9] —- Steady solutions of the Boltzmann equation for a gas flow past an obstacle, I. Existence, Arch. Rat. Mech. Anal. 84, (1983) 249-291. 